\title{
Constitutive Model for Large Strain Deformation of Semicrystalline Polymers ${ }^{\dagger}$
}

D. W. Holmes (david.holmes1@jcu.edu.au), J. G. Loughran and H. Suehrcke

School of Engineering, James Cook University, Townsville, Australia

May 15, 2007

\begin{abstract}
A constitutive model for large strain deformation of semicrystalline polymers has been formulated to predict the complex elasto-viscoelastic-viscoplastic material response. The general form of this model can be represented by three parallel rheological components corresponding to each of the modes of deformation. It will be shown that such a configuration is well suited to the mechanical nature of polymers as observed in recent studies. The constitutive stress-strain-time relationships are drawn from continuum mechanics which are more suitable than simple linear expressions from rheology. The result is a large strain, fully threedimensional constitutive model, derived from a thermodynamic basis. The proposed model can be fit to macroscopic experimental data and is highly suited to numerical analysis. The paper reviews the literature relevant to constitutive representation of semicrystalline polymers, provides conclusion and validation of the most suitable form of constitutive model and presents the relevant constitutive mathematics.
\end{abstract}

Keywords: Elasto-viscoelastic-viscoplastic, Semicrystalline Polymers, Constitutive Mathematics, Rheological Model

\section{Introduction}

Semicrystalline polymers are used widely in the manufacturing industry. Their ease of moulding and ability to be recycled makes them a very attractive material to use in a multitude of different applications, including in more recent years, where high or structural loading is expected during service. When high loads and elevated temperatures are involved it becomes increasingly important to be able to predict the loaded response of a material so as to allow confidence in service life and informed design choice. A constitutive model is a tool for such prediction and research to find an accurate constitutive model to represent semicrystalline polymers has been documented over many years by many authors.

As will be reviewed in the sections to follow, many different types of constitutive models have been proposed for application to semicrystalline polymers with varying degrees of accuracy over a variety of

$\dagger^{\dagger}$ Preprint version, the original publication is available at www.springerlink.com (C) 2007 Kluwer Academic Publishers. Printed in the Netherlands. 
different loading circumstances. To date, however, there is still no one model that is generally accepted to perform satisfactorily over a practical range of loading circumstances. This is mainly due to the complexity of the response under load of the material. As will be shown, semicrystalline polymer response comprises certain degrees of time variant and invariant response and it is the accurate account of these that has been the aim of the majority of previously proposed constitutive models. It could be argued that for the general case, this goal is yet to be achieved.

This paper partly acts to review literature associated with the development of a constitutive model for semicrystalline polymers and to collate the resulting requirements of such a model so as to allow informed selection. This paper then uses this review to justify the selection of a continuum mechanics model that has not previously been suggested for semicrystalline polymers. This model is theoretically compared with experimental testing on semicrystalline polymers from the literature as means of validation.

Section 2 provides background on constitutive modeling, specifically rheological models. In Section 3, a review of literature relevant to the aims of this paper is carried out, concluding on the requirements of a suitable model for semicrystalline polymers based on the reviewed literature. A new model is then proposed. Section 4 presents the validation of the chosen model based on theoretical comparison between model capabilities and experimental test results from the literature. The constitutive mathematics that accompany the chosen model are collated in Section 5.

\section{Background}

In continuum mechanics there are various forms of constitutive theories that can be used for representation of the deformation behavior of materials. Perić and Owen [48] outline two major model types as micromechanical models and phenomenological models. Micromechanical models predict material response by simulating interactions on a molecular level. Phenomenological theories rely on macroscopic observations of representative volumes of material. Perić and Owen make note that the most powerful phenomenological theories should still take into account the "... underlying microscopic dissipation mechanisms" (Perić and Owen [48]: 1507). One of the most common types of phenomenological theories involves using a rheological model as the frame work for a constitutive theory. Such models will be the main focus of this paper.

MTDM_revision.tex; 15/05/2007; 8:55; p.2 
Rheological models are an effective tool for understanding and representing the mechanical properties of a material. By combining simple elements such as springs and dashpots, a complicated material response to loading can be represented by a combination of easily visualized simple responses. In this form a rheological model constitutes a constitutive theory. In deformation analysis, rheological constitutive theories are widely used for two main purposes. The first of these is as a conceptual aid to help visualize the way in which a material behaves during deformation. An example of this would be to visualize the behavior of a spring when trying to understand elastic material behavior. The second is as a quantitative tool for use in predicting the loaded response of a material either manually or numerically in the form of a finite element simulation. The second of these is more pertinent to our endeavor.

When used in the form of a quantitative constitutive theory, rheological models provide the framework to which controlling constitutive mathematics are applied. Such mathematics can be either derived from the linear component expressions such as Hooke's laws in the case of a spring or from analogous but more complicated, nonlinear theories. Such nonlinear theories can take direction from micromechanical dissipation (Perić and Owen [48]). Where the linear constitutive expressions allow simplicity of formulation and implementation, the nonlinear theories allow for, in many cases, a more realistic representation of loaded responses where complicated load and rate dependencies are involved. The constitutive mathematics dictate the relationships between stress and strain as well as other parameters including strain-rate, time, temperature and material variables for each rheological component.

The configuration of a rheological model governs the interrelationships between each element. Different positioning of springs, dashpots, etc. in a rheological model can result in very different model responses to deformation. The presence and timing of required forms of deformation can be controlled by the suitable selection of model configuration. It is equally important to have both a well constructed rheological framework and plausible constitutive mathematics.

Choosing the correct rheological configuration accompanied by constitutive mathematics that adequately account for any required dependencies can result in a very powerful constitutive theory. Semicrystalline polymers are a complex material, as will be demonstrated, and so it is the aim of this paper to select and validate the best constitutive theory for semicrystalline polymers based on previous research.

MTDM_revision.tex; 15/05/2007; 8:55; p.3 
Holmes et al.

\section{Review of Literature}

Given the shear volume of published research on the constitutive behavior of semicrystalline polymers, within the current investigation it would seem impractical to provide a comprehensive review of the literature. Rather, it is the aim here to enable conclusion on the requirements of a general model for semicrystalline polymers and to report on a representative cross-section of constitutive model types that have been used to date. The survey allows informed specification of the most appropriate model for this type of material.

\subsection{DireCtion From Micromechanics}

The molecular deformation mechanisms treated by micromechanical constitutive theories are generally measured explicitly during observation on molecular interactions using techniques like X-ray scattering [18]. Such testing is significantly more definitive in regards to measuring the actual modes of deformation present (elasticity, viscoelasticity, etc) than the global stress-deformation measurement techniques used in macroscopic research. Correspondingly, while not the primary focus of this investigation, a brief review of micromechanics would seem beneficial.

Some of the most comprehensive work on semicrystalline polymer micromechanical modeling can be attributed to Drozdov and co workers (see for example Drozdov and Christiansen [11] for polypropylene and Drozdov and Yuan [14] for low density polyethylene). Like some of Drozdov's similar work with amorphous polymers [9, 13], this work reports on an elasto-viscoelastic-viscoplastic constitutive model that functions by mimicking molecular chain interactions to predict small strain deformation. In the small strain region, fair agreement between model prediction and experimental observation is reported. Unfortunately, because semicrystalline polymers have highly anisotropic microstructures [10], while generally exhibiting isotropic deformation characteristics on the macroscopic scale (given ideal moulding conditions), extension to macroscopic applications is problematic and consequently shown to be inaccurate. This type of scaling discontinuity and the numerical cost due to the complexities associated with implementation on a practical scale, mean a model such as this is inappropriate for our purposes. The limitations of micromechanical models however, in no way detract from the significance of actual molecular level deformation measurement. This will be the focus of the remainder of this subsection.

MTDM_revision.tex; 15/05/2007; 8:55; p.4 
Firstly, it is important to note that semicrystalline polymers are characterized by a morphology of crystalline regions of ordered lamellae, surrounded by amorphous regions of unordered molecular chains. The amorphous regions in close proximity to, or between crystalline lamellae are noted to have greatly reduced mobility and are often regarded as a third region $[5,10,11]$. It is the interactions and behavior of these structures that account for the complex deformation behavior of semicrystalline polymers.

It is widely accepted that on the molecular level, deformation of the semicrystalline structure results from a combination of elastic, viscoelastic and viscoplastic modes (see for example [11, 12, 14]). Elasticity is associated with the stretching of molecular chains within the amorphous phase $[5,18]$. A mechanism that can be compared to the behavior of purely hyperelastic materials such as rubbers and elastomers [40]. Drozdov and co workers $[10,11,12,13]$ conclude that viscoelastic response is the result of molecular chain rearrangement within the amorphous phase; primarily, the separation of chains from lamellae junctions and connection of free chains with new junctions. Thermal activation is thought responsible for these rearrangements where an increase in a molecules energy enables its jump to a higher state [10]. The concept of viscoelastic recovery is then viewed as the progressive return of the molecular chains to their original state upon the removal of energy from the system (i.e. unloading). Viscoplastic deformation has been observed by Schrauwen et al. [57] and also Drozdov and Christiansen [12] to initiate in the amorphous region via interchain slippage ${ }^{1}$. At higher strains, this transitions into the crystalline phase where fine, and then coarse lamellae sliding occurs with eventual fragmentation of the crystallites.

An explicit yield threshold is commonly observed at small strain levels (for example $\varepsilon=0.02$ [11]) far before necking. Schrauwen et al. [57] have also observed additional yielding phenomena for strains in excess of necking (up to $200 \%$ strain). The actual mechanisms of yield are noted to be the source of some conjecture [11], however generally, yield results from the increased load on molecular chains (most significant in the crystalline phase [57]), where some required threshold of thermodynamic potential is reached so as to allow slippage of chain-chain bonds. Strain hardening is also commonly observed. G'Sell et al. [18] explains such phenomena to be primarily the result of lamellae fragmentation that corresponds to an increase in the restricted amorphous phase. This phase reinforces the remaining lamellae fragments and so limits further

\footnotetext{
${ }^{1}$ Providing temperature is below the glass transition temperature such that the amorphous phase is in glassy state.
}

MTDM_revision.tex; 15/05/2007; 8:55; p.5 
viscoplasticity. The chain density in the amorphous phase before and after deformation plays a key role in this phenomena [57].

A fully general macroscopic model should account for all the deformation mechanisms observed within the microstructure, as summarized here.

\subsection{Macroscopic Constitutive theories}

The review that follows is concerned mainly with large strain constitutive models for semicrystalline polymers. Based on the conclusions from the preceding section, our attention will be limited to models that account for some amount of elastic, viscous and plastic deformation.

Findley et al. [17], while largely concerned with nonlinear viscoelastic integral theories, presents a simple elasto-viscoelastic-viscoplastic rheological model termed the Burgers model (Figure 1 (a)). While the Burgers model can reproduce the trends of some polymer experimental curves, it has been discounted mainly due to the fact that polymer viscous and plastic responses are known to be nonlinear for all cases other than for small strain. Findley et al. [17] summarized a shift from the use of conventional rheological models due to their restriction of linearity. Modern rheological models can be used as framework for nonlinear theories, however the other limitation to the Burgers model is its lack of expression for an actual yield threshold with irrecoverable deformation being present at all stages of loading.

G'Sell and Jonas [21], Kitagawa and co-workers [31, 32, 33] and Schang et al. [54] all made contributions toward validating an elastoviscoelastic-plastic model for application to a wide range of semicrystalline polymers including polyethylene, polypropylene and polyamide 12. The model used was derived from the governing constitutive equation for a three element viscoelastic rheological model, adding components to the expression to incorporate nonlinearity and plasticity. The addition of nonlinearity and plasticity was done mathematically and so the end model was no longer analogous to any rheological configuration. Whilst the authors of the above papers note their theory to be elasto-viscoelastic-plastic, the overall response is represented via a single governing constitutive equation. The fit of this theory to experimental data would thus account for the required components of elastic, viscous and plastic deformation automatically without explicit definition of each actual mode of deformation. This can prove to be a weakness with single expression theories derived directly from curve fitting because extrapolation outside the ranges of testing can diverge very rapidly from a reasonable prediction in part due to the nature of polynomial type fitting. In spite of this weakness, the model was tested

MTDM_revision.tex; 15/05/2007; $8: 55 ;$ p.6 


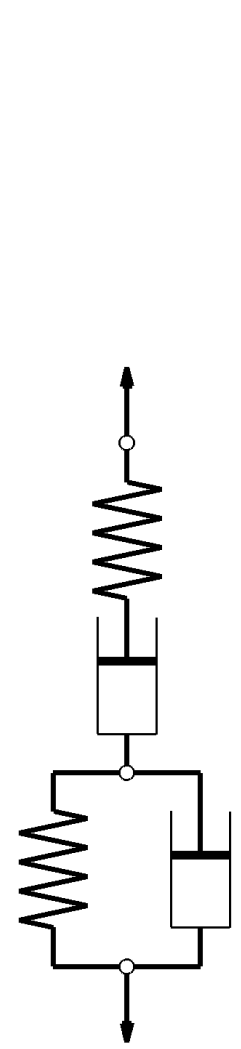

(a)

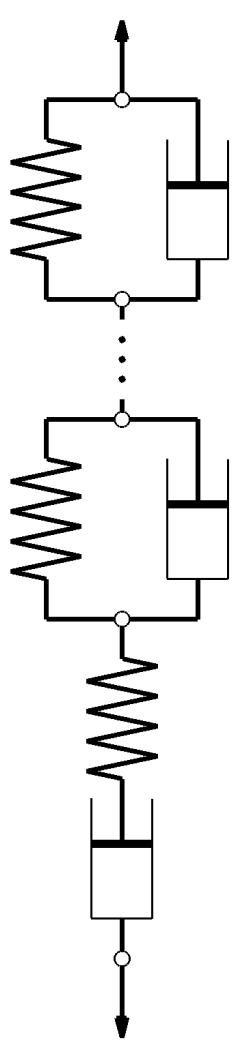

(b)

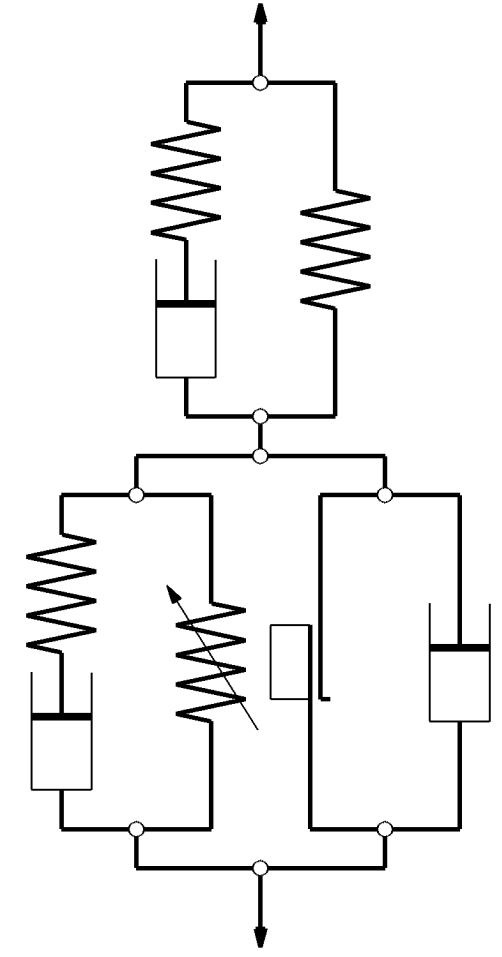

(c)

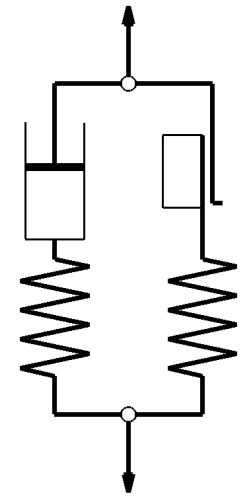

(d)

Figure 1. One-dimensional elasto-viscoelastic-viscoplastic rheological models used for semicrystalline polymers by (a) Findley et al. [17], (b) Schapery [55], (c) Brusselle-Dupend et al. [5, 6] and (d) Kletschkowski et al. [34].

over a variety of different loading cases and was shown to reproduce the experimental results well for all tested cases of positive strain, well into the necking region. The model was shown in all cases, however, to be inaccurate during unloading.

Lai and Bakker [36] propose an elasto-viscoelastic-plastic integral form constitutive theory for high density polyethylene. Total strain is additively decomposed into viscoelastic and plastic components and the constitutive theory is fulfilled using data from creep and recovery tests. The theory is shown to be accurate for simulation of various creep, recovery and constant stress rate loading and unloading. Again the model is phenomenological in nature but has little to do with actual molecular behavior.

Schapery $[55,56]$ proposes a nonlinear elasto-viscoelastic-viscoplastic constitutive model derived from thermodynamics. While these papers 
are largely theoretical the first is written with the aim of representing fibre reinforced and un-reinforced plastics while the second is more generally noted to apply to several materials including semicrystalline polymers. Schapery's model is based on the theoretical rheological framework shown in Figure 1 (b). This model is similar to the Burgers model shown in Figure 1 (a) however it makes provision for any number of viscoelastic components as may be required and accounts for all constitutive relationships in a nonlinear fashion. These component constitutive relations are based on thermodynamics rather than linear rheology. Schapery discusses theories to incorporate various additional nonlinearities, as well as other possible viscoplastic theories that may also incorporate yield.

Zhang and Moore [64, 65] report on the elasto-viscoelastic-viscoplastic behavior of high density polyethylene pipes across constant strain rate, transient strain rate, single and cyclic loading-unloading, creep, relaxation and combination tests. They then propose a nonlinear elastoviscoelastic model derived from nonlinear rheology and an elasto-viscoplastic model derived from works by Bodner and Partom $[1,2]$ on steel, to represent observations. Both are uniaxial theories and the elasto-viscoplastic model assumes viscoplastic deformation to occur at all times and thus no yield expression is required. The two theories were compared against experimental results. It was observed that the elasto-viscoplastic theory was the superior of the two, closely predicting the majority of results seen experimentally. The nonlinear viscoelastic model was shown to be weak in predicting rapid changes in applied strain or strain rate while the only weakness with the elasto-viscoplastic model was its inability to predict strain reversal. While it would seem each of the tested models have certain advantages, it could be concluded that a more generally applicable model would incorporate both components of viscoelasticity and viscoplasticity. Other limitations with the models include the uniaxial restriction and the lack of expression for actual yield threshold or hardening in the elasto-viscoplastic model.

One of the more comprehensive testing procedures and constitutive models for semicrystalline polymers to date was published in two parts by Brusselle-Dupend et al. [5, 6]. They propose a uniaxial testing regime for polypropylene in the pre-necking region, designed to isolate the elastic, viscoelastic and viscoplastic components of deformation. Preliminary tests showed this pre-necking region to be within $11 \%$ strain for polypropylene. The authors proceed to use the test data to validate an elasto-viscoelastic-viscoplastic constitutive model based on the rheological framework shown in Figure 1 (c). The proposed model contains a viscoelastic component in series with a viscoplastic component. During testing the authors observe both kinematic and 
isotropic hardening and account for this in the rheological framework of the viscoplastic element. During tests at low strain, Brusselle-Dupend et al. also report a transitional zone between purely elasto-viscoelastic response and elasto-viscoelastic-viscoplastic response to occur at approximately $1.5 \pm 0.3 \%$ strain. Thus the material was seen to have a definite viscoplastic yield point which was also accounted for in the model.

The viscosity of semicrystalline polymers (dash pot coefficients, Figure 1 (c)) have been shown by Eyring [16] and later Halsey et al. [29] to evolve with varying inelastic strain rate (the Eyring model). BrusselleDupend et al. $[5,6]$ found in experimentation that the Eyring model for evolution of viscosity, for each of the viscoelastic and viscoplastic flows was inadequate and so new expressions were formulated with added dependencies on total strain rate. The new viscosity expressions were shown by comparison to be highly superior to the polymer viscosity models used previously.

Constitutive model validation for the Brusselle-Dupend model was carried out by comparison with experimental data and it was shown that the simulations closely reproduced the expected results for multiple cases of loading including combinations of loading, unloading, relaxation and recovery for several cases of strain rate. The work of Brusselle-Dupend and co-workers makes one of the most comprehensive attempts at accounting for the many mechanisms of semicrystalline polymers during deformation. While their model proved accurate across all cases published, there are still some important limitations. The rheological configuration is relatively complex especially the representation of strain hardening. The formulation of the model is largely phenomenological and, while accurate in application, it takes no direction from actual thermodynamics of the material continuum. Finally one of the more major limitations is that the theory is formulated for uniaxial application and would require significant work to extend the theory to account for three-dimensions.

Recently Kletschkowski et al. [34] presented a simple rheological constitutive model to represent the response of semicrystalline polytetrafluorethylene, (Figure 1 (d)). From the configuration of the rheological model, it can be seen to sequentially account for viscoelasticity, then viscoplasticity within the same viscous element, the transition being governed by the state of yield. Throughout validation with experimental testing, Kletschkowski demonstrates a reasonable correlation between model predictions and the results for polytetrafluorethylene. While demonstrating some correct predictions, it could be concluded that for many cases this type of model is oversimplified. As has been found in many of the reviews above, viscoelastic and viscoplastic de- 
formation is know to occur simultaneously, however Kletschkowski's model accounts for these modes sequentially and so can be deemed to be questionable for the general case.

Perić and Dettmer [46] presented a finite strain constitutive theory generally applicable to most types of materials. Their model encompasses any combination of elastic, viscoelastic, elasto-plastic or viscoplastic response. Simultaneous account of these deformation modes is made possible by the parallel rheological framework on which the model is based. The generalized one-dimensional rheological representation is shown in Figure 2. The accompanying constitutive mathematics are derived from thermodynamics, allowing for both plastic and viscoplastic yield. Isotropic hardening is touched on and it is possible to extend the theory to account for kinematic hardening also, following references such as Simo [61]. The technique of using continuum mechanics theories implemented over a rheological framework has found wide prior application in specific forms for large strain deformation of inelastic materials (mainly rubbers and steels) as in works by Simo [58, 59], Simo and Hughes [60, 61], Perić and Owen [47, 48], Reese and Govindjee [52] and Rosati and Valoroso [53]. Perić and Dettmer show that in a very general sense a model of this type can be widely generalized via the parallel combination of all the different types of possible elements (elastic, viscoelastic, viscoplastic and elasto-plastic). Perić and Dettmer demonstrate the models versatility by presenting numerical simulations completed using elasto-viscoelastic and elasto-viscoelastic-plastic forms of the model, each sited as representing the response of various types of rubber ${ }^{2}$.

The works of Nedjar $[38,39]$ deal with the formulation and numerical implementation of two large strain continuum mechanics type theories for application to filled rubbers, polymers and polymeric foams. The two different formulations are presented together and compared. The first model is based on a parallel combination of viscoelastic and viscoplastic rheological elements identical to a reduced form of the Perić and Dettmer model, Figure 2. The second model is based on a series rheological addition of viscoelastic and viscoplastic components, similar to that used by Brusselle-Dupend et al. shown in Figure 1 (c). Like the work of Perić and Dettmer, both Nedjar's models are formulated for three-dimensional, large strain with constitutive mathematics developed from thermodynamics. Again, only linear viscosities are used. The second series model treated, involves no account of strain hardening and it would seem from the work presented that such a configuration is inappropriate for our purposes from both a response and implemen-

\footnotetext{
${ }^{2}$ Only constant viscosity coefficients are used in these studies.
} 


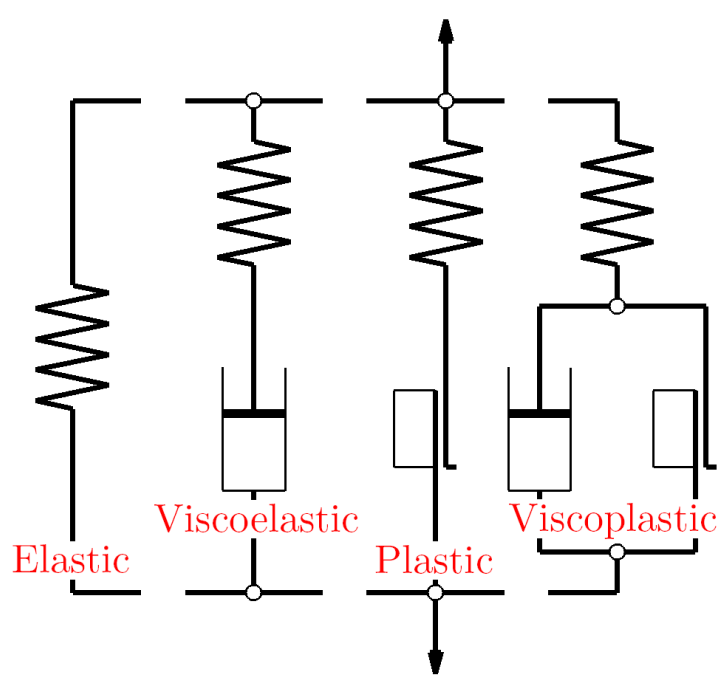

Figure 2. Generalized one-dimensional rheological representation of the elasto-viscoelastic-plastic-viscoplastic Perić and Dettmer [46] model.

tation stand point. The first parallel model, however, does incorporate isotropic hardening (and as before, kinematic hardening could be included with some development) and would seem an attractive model for semicrystalline polymers. A downfall of the specific model of Nedjar is, however, the lack of a wholly elastic component of response as was included in the Perić and Dettmer form. It will become evident in Section 4 , that such an element is important for the response profiles observed during unloading and recovery, as observed by Brusselle-Dupend et al. $[5,6]$ and so a reduced form of the model of Peric and Dettmer would seem the more appropriate choice as will be further discussed in the next section.

\subsection{Model SELECTION}

It has only been in the last few decades that the technology has been available to allow microscopic deformation analysis. The great majority of macroscopic constitutive theories (dating back to the 1960s) were formulated based solely on experimental observation and phenomenology, thus limiting the ability to independently distinguish actual deformation modes. This perhaps explains the length of time it has taken for this type of research to come to the conclusions that were confirmed so comparatively quickly by micromechanics.

Referring to the most recent works by Zhang and Moore [64, 65] and Brusselle-Dupend et al. [5, 6], the micromechanical observations that semicrystalline polymers behave elasto-viscoelasto-viscoplastically 


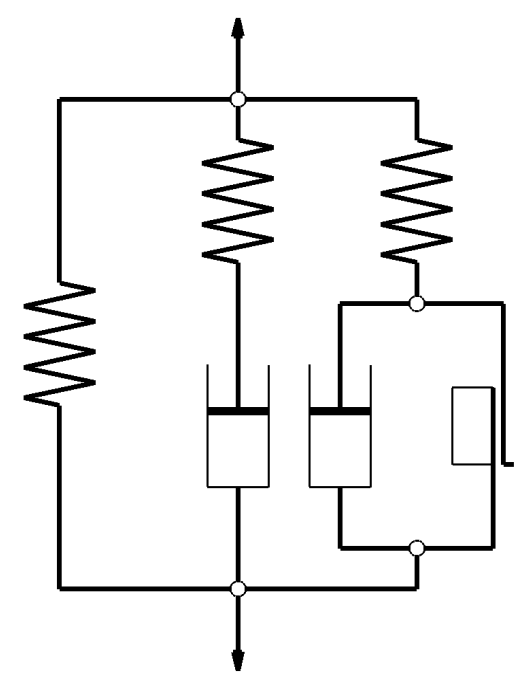

Figure 3. One-dimensional elasto-viscoelastic-viscoplastic rheological model.

with viscoplastic yield and strain hardening, have all been confirmed at macroscopic scale. In addition, macroscopic strain hardening has been observed to occur via both isotropic and kinematic mechanisms $[5,6$, $18,19,22,57]$ while a significant strain rate dependence of both the viscoelastic and viscoplastic viscosities has been observed $[5,6,16,29]$. No one existing model accounts for all these required behaviors and so an appropriate combination of components from existing theories will be used.

Based on the required deforming characteristics, we propose that a modified elasto-viscoelastic-viscoplastic form of the Perić and Dettmer model (Figure 3) be used, incorporating both isotropic and kinematic hardening as well as nonlinear viscosity expressions comparable to those presented by Eyring [16] and Brusselle-Dupend et al. [5, 6].

A major advantage of this type of model is associated with the origins of the constitutive mathematics. While not explicitly modeling the micromechanics of deformation, continuum mechanics theories such as this are based on thermodynamic laws that represent the global effect of molecular interactions. Thus, direct analogy between such theories and the mechanisms discussed in Section 3.1 is possible.

Firstly, hyperelastic theory is used for the elastic element where the energy of deformation is related to the thermodynamic system free energy in a similar way to that used in the micromechanical theories [11]. For viscoelasticity, viscous evolution is related to thermodynamic dissipation $^{3}$ and is initiated and driven via interaction with another

\footnotetext{
${ }^{3}$ Satisfying the Clausius-Duhem form of the second law of thermodynamics [52]
} 
hyperelastic element, as would be the case on the molecular level. The viscoplastic evolution is also related to thermodynamic dissipation and is initiated by loading originating from elasticity. Isotropic and kinematic hardening are included which represent the fragmentation and subsequent fibril reinforcing of crystalline lamellae. For deformation levels up to necking, a single yield point (well below the onset of necking) represents where microscopic yielding mechanisms become macroscopically evident.

The intimate relationship between macroscopic representation and the actual microscopic deformation mechanisms, further reinforces the value of a model such as this.

\section{The Suitability of the Selected Rheological Form to Polymers}

While the chosen Perić and Dettmer parallel model configuration (Figure 3 ) is desirable from a numerical standpoint, its explicit applicability to semicrystalline polymer response is yet to be confirmed. As a preliminary assessment for the performance of the parallel rheological configuration, one-dimensional, linear constitutive mathematic from rheology were used ${ }^{4}$. Hooke's law was used for the elastic element(s) $[17]$

$$
\sigma_{e}=E_{e} \varepsilon
$$

and a simple newtonian evolution expression was used for the viscoelastic element [17]

$$
\sigma_{v e}=E_{v e}\left(\varepsilon-\varepsilon_{v e}^{i}\right), \quad \dot{\varepsilon}_{v e}^{i}=\frac{1}{\eta_{v e}} \sigma_{v e}
$$

Further a one-dimensional form of the Perzyna model equations were used for viscoplasticity (refer to Appendix B for further details)

$$
\begin{gathered}
\sigma_{v p}=E_{v p}\left(\varepsilon-\varepsilon_{v p}^{i}\right), \quad \dot{\varepsilon}_{v p}^{i}=\frac{\langle f\rangle}{\eta_{v p}} \frac{\partial f}{\partial \sigma_{v p}} \\
f=\left|\sigma_{v p}\right|-\sigma_{y}
\end{gathered}
$$

The material parameters were chosen arbitrarily and are presented for completeness in Table I.

The viscoelastic and viscoplastic evolution equations were solved using a Newton iteration scheme, analogous to that presented by Reese

\footnotetext{
4 The actual nonlinear, three-dimensional constitutive mathematics are treated at length in Section 5.
} 
Table I. Arbitrarily chosen 1D linear model parameters

\begin{tabular}{lrl}
\hline$E_{e}$ & {$[\mathrm{MPa}]$} & 250 \\
$E_{v e}$ & {$[\mathrm{MPa}]$} & 1000 \\
$E_{v p}$ & {$[\mathrm{MPa}]$} & 1000 \\
$\eta_{v e}$ & {$[\mathrm{MPa} \mathrm{s}]$} & 1000 \\
$\eta_{v p}$ & {$[\mathrm{MPa} \mathrm{s}]$} & 1000 \\
$\sigma_{y}$ & {$[\mathrm{MPa}]$} & 1 \\
\hline
\end{tabular}

and Govindjee [52]. A simple strain controlled computational implementation was used to generate the data presented in what follows. By showing that the parallel configuration is capable of reproducing the same deformation characteristics observed in the literature, the configuration is proven valid for these purposes.

\subsection{Static LoAding}

Some of the earliest tests on the time dependent nature of polymers were static creep-recovery tests; Onaran and Findley [42], Lifshitz and Kolsky [37] and others as summarized by Findley et al. [17]. These tests involved maintaining a constant stress on a test sample and observing the deformation response. Load removal then results in elastic and some degree of time dependent strain recovery. This observed deformation response can be effectively reproduced by the 3 element, elasto-viscoelastic-viscoplastic model (Figure 3). During loading the applied stress is distributed through the three elastic springs with the viscoelastic and viscoplastic component springs driving time dependent deformation in the corresponding dashpots (providing spring stress exceeds that of yield in the case of the viscoplastic element). When an applied stress is held constant the evolution of the viscoelastic and viscoplastic dashpots proceeds while their element stresses decrease to zero and below yield respectively while the elastic element stress increases to compensate and maintain a constant total load. The increasing elastic element stress necessitates an increase in total deformation and this time dependent strain increase is referred to as creep. If viscoplastic yield is not reached during initial load application, the element will act as an additional elastic element with an analogous increase in stress. In this way, viscoplastic yield can either be achieved by an initially large applied stress, or as a result of increasing creep strain, inducing increased stress in the viscoplastic spring until yield 
is reached. This property effectively reproduces the phenomenon of plastic residual strain being present in polymer samples following long term creep testing at stresses far below the expected yield (Findley et al. [17]).

Release of the load results in a total model stress of zero, however due to dashpot strains the most likely stress distribution in the springs is one of tension in the elastic element and a counteracting compression in the other two. This residual element stress is what drives the creep mechanism in reverse, referred to as recovery. While the viscoelastic element stress will proceed to zero, that in the viscoplastic element will stop when the element stress decreases below compressive yield stress. As such a residual strain remains in the model. These results are all comparable to those observed by Onaran and Findley [42], Lifshitz and Kolsky [37] and Findley et al. [17] for actual experiments on semicrystalline polymers.

Strain control tests are a powerful type of test for formulating and testing a material with simultaneous modes of deformation such as is the case for semicrystalline polymers. Such testing simplifies the isolation of the parallel modes of deformation and has been used almost exclusively in more recent polymer testing. Relaxation testing is one of the earliest forms of this type of test. A relaxation test involves maintaining a constant strain on a test sample and observing the response to stress (Findley et al. [17]).

The response of the proposed model (Figure 3) during stress relaxation can again be audited. The initial strain application will result in a distribution of the total stress across the three element springs driving inelastic evolution in the case of the viscoelastic and viscoplastic elements in a similar fashion as above. Upon hold of the level of strain, the elastic element stress will remain constant while that in the viscoelastic and viscoplastic elements will decrease with the progressive evolution of the dashpots. This progressive decrease in stress is referred to as stress relaxation. Again the viscoelastic stress will approach zero while the stress in the viscoplastic element will become constant when the spring stress becomes less than that of yield. Once again this type of response is exactly as would be expected for a semicrystalline polymer as reported by Findley et al. [17].

\subsection{Constant Strain Rate Loading}

Constant strain rate loading is one of the most common testing methods used for the evaluation of polymer constitutive theories. A constant strain rate test involves a linear ramp in strain over time. Figure 4 (a) shows a constant strain rate test simulation over time on a proposed 


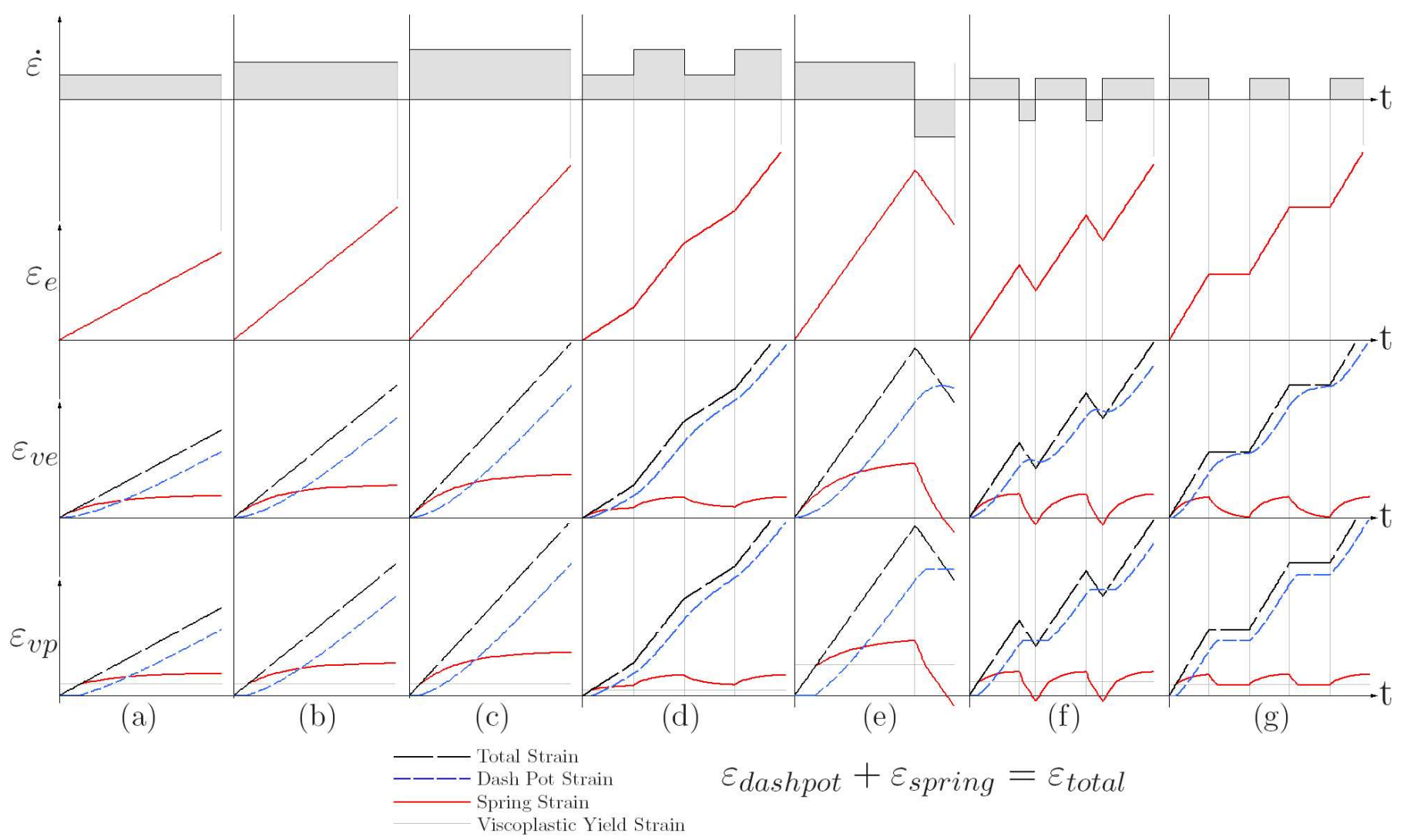

Figure 4. Theoretical strain curves for the proposed elasto-viscoelastic-viscoplastic rheological model showing all element components of strain for ( $\mathrm{a}$, b and c) constant strain rate tests, (d) changing strain rate tests, (e) loading-unloading test, (f) cyclic loading-unloading test and $(\mathrm{g})$ cyclic loading-relaxation test. Figures are not drawn to scale.

rheological model configuration. Four graphs are shown; the top is the magnitude of strain rate over time followed by component strain vs. time response for the elastic, viscoelastic and viscoplastic elements respectively. The strain vs. time graphs show the total element strain (all three elements have equal total strain), the component of strain in the element spring and the component of strain in the dashpot is as per the key shown. Spring and dashpot strain components are a decomposition of the total strain for each element. For the viscoplastic element response, a faint horizontal line shows the spring strain corresponding to the element yield stress.

Figure 4 can be used to demonstrate the relative contributions to stress of each model component where stress is linearly related to spring element strain in each case. Figure 4 (a), (b) and (c) are representative 
of three different strain rates applied to the same model. It can be observed that an increase in strain rate corresponds directly to an increase in each component's level of spring strain and so total stress. Total model stress has been calculated and is plotted against total strain in Figure 5 (a) for these three strain rates. Authors such as G'Sell and Jonas [20, 21], Kitagawa and colleagues [31, 32, 33], Popelar et al. [50], Duffo et al. [15], Zhang and Moore [64, 65] and Kontou and Farasoglou [35] have readily observed constant strain rate tensile test curves such as those in Figure 5 (a). One such experimental curve set is shown in Figure 6 (a) ${ }^{5}$ for comparison (taken from Kitagawa et al. [33]). Similarly shaped curves and similarly increasing levels of stress corresponding to the increasing strain rates are observed by all authors and can be likened to that seen here for the proposed elasto-viscoelastic-viscoplastic model.

Tests that are often carried out during polymer research involve constant strain rate tests with mid test transient jumps to different values of strain rate. A theoretical simulation of such a test has been carried out for the proposed rheological model in a similar fashion to the constant strain rate tests above. Figure 4 (d) shows the theoretical test strain rate, elastic, viscoelastic and viscoplastic component strains against time. It can be observed that for all elements, modifications in strain rate affect all components of strain as if the difference in strain rate has been added to or subtracted from the existing response. It can also be observed that the spring strain for the viscoelastic and viscoplastic elements always approach the same equilibrium value for the same applied strain rate. Again, the total model stress response is shown against total strain in Figure 5 (b). The monotonic paths corresponding to each strain rate are superimposed on the figure. The observed path of the stress strain curve due to transient changes in strain rate and the apparent lack of history dependence on strain rate has been observed in experimental testing on semicrystalline polymers by G'Sell and Jonas [21], Kitagawa and Matsutani [31], Kitagawa et al. [32], Kitagawa and Takagi [33] and Zhang and Moore [64, 65]. Experimental results from Zhang and Moore [64] are shown in Figure 6 (b) for comparison.

Other types of tests using changing states of constant strain rate have also been used in the evaluation of polymer constitutive models. Kitagawa and Matsutani [31], Popelar et al. [50], Zhang and Moore [64, 65], Pegoretti et al. [44], and Brusselle-Dupend et al. [5, 6] have all conducted loading-unloading tests where both the loading and unloading

\footnotetext{
${ }^{5}$ Figure 6 (a) to (d) Reproduced with kind permission of John Wiley \& Sons, Inc.
}

MTDM_revision.tex; 15/05/2007; 8:55; p.17 


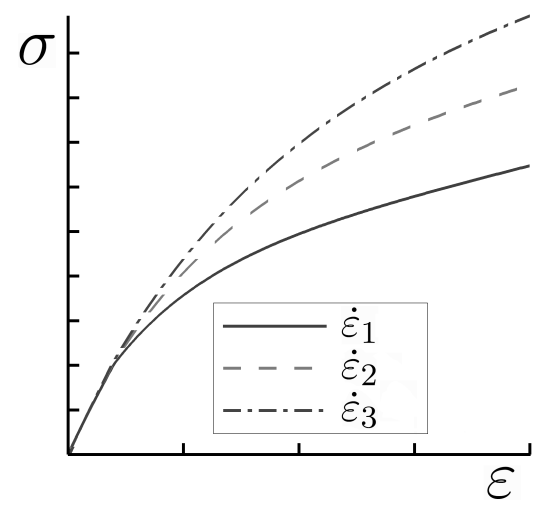

(a)

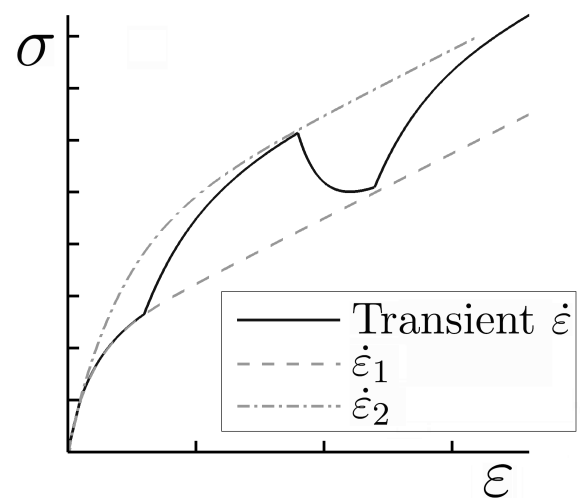

(b)

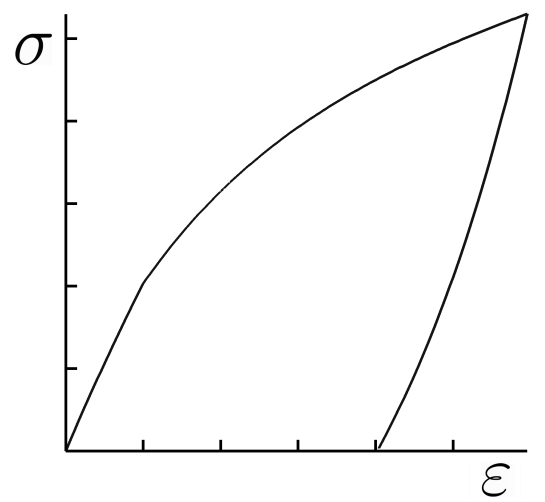

(c)

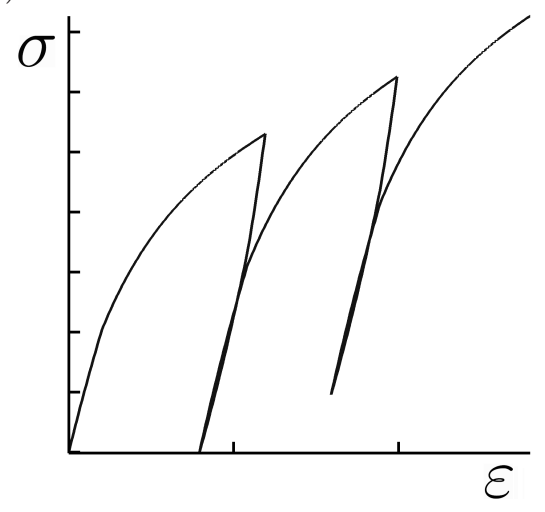

(d)

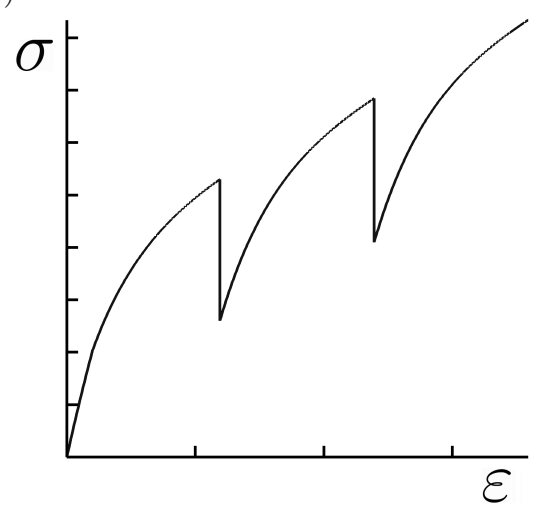

(e)

Figure 5. Theoretical total stress vs. total strain curves for the proposed elasto-viscoelastic-viscoplastic rheological model for (a) three constant strain rate tests, (b) changing strain rate test, (c) loading-unloading test, (d) cyclic loading-unloading test and (e) cyclic loading-relaxation test. Figures are not drawn to any scale.

are conducted at a constant strain rate of the same magnitude but opposite sign. Figure 4 (e) shows the proposed model theoretical strain component results. The total theoretical stress in the model has been calculated, as before, and is shown against total strain in Figure 5 (c). It is worth noting that the unloading carried out has ended at a point of zero stress, while there is still a large degree of strain. This property and the shape of the loading and unloading stress vs. strain curves are analogous to the observations from experiments of the aforementioned authors; Figure 6 (c) is indicative of such results for polypropylene (Brusselle-Dupend et al. [6]). As before, the driving mechanism behind recovery is the residual compressive strains left in the viscoelastic and viscoplastic elements as can be seen in Figure 4 (e). 


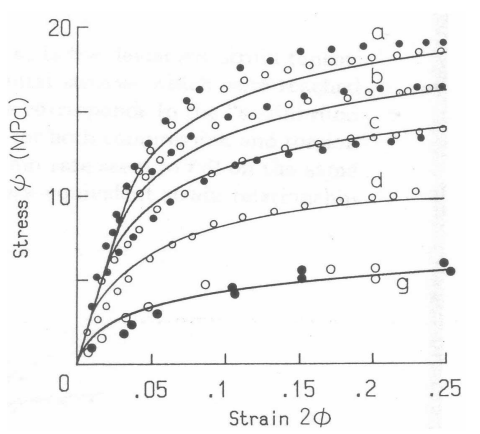

(a)

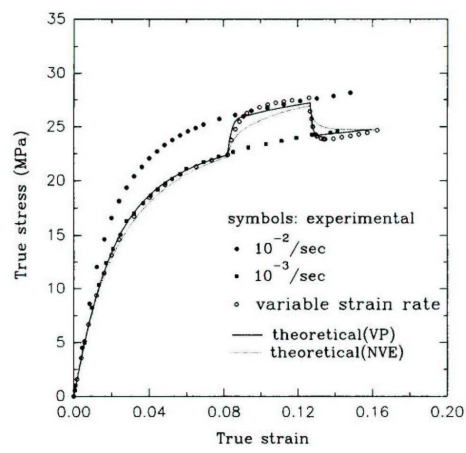

(b)

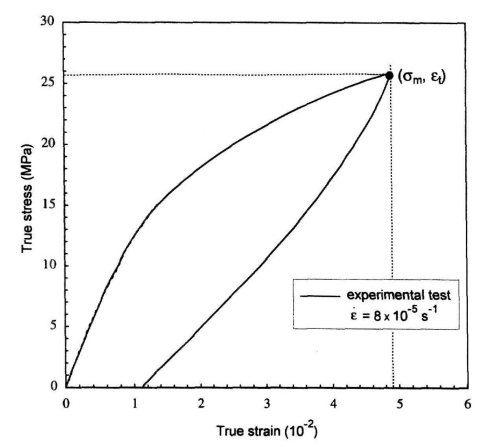

(c)

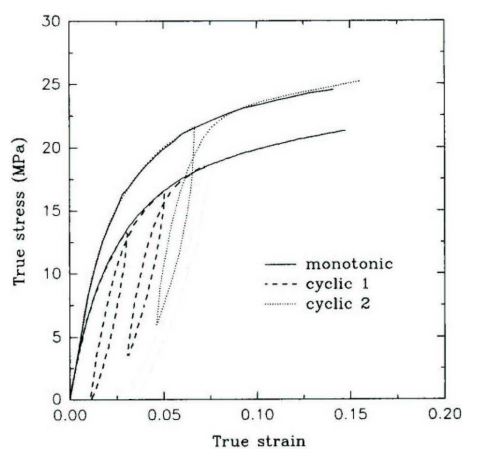

(d)

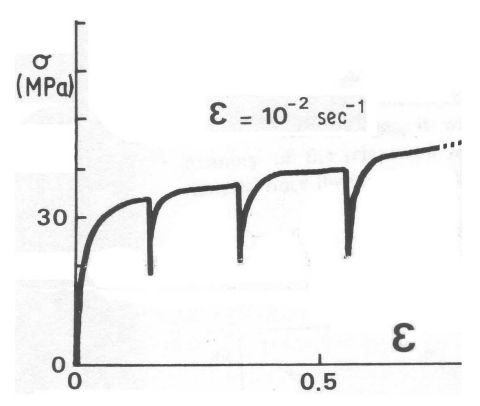

(e)

Figure 6. Experimental total stress vs. total strain curves taken from the literature for (a) constant strain rate tests, Kitagawa et al. [33]: Figure 4, p 1947, (b) changing strain rate test, Zhang and Moore [64]: Figure 3, p 407,(c) loading-unloading test, Brusselle-Dupend et al. [6]: Figure 13, p 512, (d) cyclic loading-unloading test, Zhang and Moore [64]: Figure 5, p 407 and (e) cyclic loading-relaxation test, G'Sell and Jonas [21]: Figure 7, p 1962 (shown to 0.75 strain).

A variation of the loading-unloading test that has been used by G'Sell and Jonas [21], Kitagawa and Matsutani [31] and Zhang and Moore [64, 65] involves cycling the loading-unloading procedure. The theoretical strain results to such a test are shown in Figure 4 (f). As was the case for the transiently changing positive strain rates in Figure 4 (d) and Figure 5 (b), the viscoelastic and viscoplastic spring strains always approach the same levels during the same strain rate. Again the total model stress has been calculated and is shown against total strain in Figure 5 (d). It can be observed that upon reapplication of the loading, the stress vs. strain curve quickly approaches the path it would have traveled were no unloading to have taken place. The shape of the reloading curve does not match that of the initial loading but rather is steeper. Both of these properties have been observed by 
the authors referenced above, an example of which is provided from Zhang and Moore [64] in Figure 6 (d) for polyethylene. Another point of comparison is that the point of stress reached by consecutive unloadings increases with each unloading instance observed in both Figure 5 (d) and Figure 6 (d).

Another form of cyclic test that is used during testing on semicrystalline polymers is cyclic loading-relaxation. This test loads the sample at a constant strain rate and then for a designated period of time, maintains constant strain, observing the relaxation in stress. This portion of stress relaxation is exactly the same as has been discussed above in the static loading section except that the level of strain was reached by a ramp in strain rather than a simple transient step. Component strains for a theoretical simulation for this type of loading are shown in Figure 4 (g). The relaxation periods are simply periods of zero strain rates as shown. The total model stress has been calculated and is shown against total strain in Figure 5 (e). Again upon reapplication of the loading, the stress-strain curve quickly approaches the path it would have traveled were there no unloading. Comparison for the initial loadingrelaxation curve component can be made to that reported for PP by Brusselle-Dupend et al. [5, 6]. The results for cyclic loading-relaxation simulations are also comparable to those found during testing by G'Sell and Jonas [21], Kitagawa and Matsutani [31] and Kitagawa et al. [32]. A figure for comparison with actual experimental result on HDPE is given in Figure $6(\mathrm{e})^{6}$ from G'Sell and Jonas [21].

\subsection{Other Phenomena}

Another property that polymers are known to exhibit is the full recovery of plastic deformation upon heat treatment. This full recovery is reported to be possible after tests of up to $20 \%$ strain in semicrystalline polymers (Pegoretti et al. [44]) and up to 50\% strain in amorphous polymers (Oleynik [41] and Quinson et al. [51]). Most importantly Pegoretti et al. [44] notes that the plastic deformation in semicrystalline poly(ethylene-terephthalate) or PET resulting from tests carried up to $20 \%$ strain at room temperature, can be fully recovered during a 6 min heat treatment at $160{ }^{\circ} \mathrm{C}$. This temperature is approximately $45^{\circ} \mathrm{C}$ higher than the materials glass transition temperature. The necking point of a polymer has long been know to be influenced by temperature (Brostow and Corneliussen [4] and Budinski and Budinski [7]) so it would be intuitive to predict that the actual viscoplastic yield would also be influenced in such a manner. With such an added dependency

\footnotetext{
${ }^{6}$ Figure 6 (e) Reproduced with kind permission of Springer Science and Business Media.
}

MTDM_revision.tex; $15 / 05 / 2007 ; 8: 55 ;$ p.20 
the proposed model would also be capable of reproducing the plastic recovery observed by Pegoretti et al. [44].

With correct expression of the temperature dependency of the viscoplastic yield stress in both tension and compression, the proposed model would be capable of total thermal plastic recovery. Referring to the earlier explanation, recovery is driven by non-zero residual element spring stresses. Following load removal, the compression in the viscoelastic component drives a full recovery of deformation. The viscoplastic element does not however recover because in most room temperature cases, the compressive yield of the material will be far greater than the residual compressive stress left in the viscoplastic component. Allowing a high enough temperature in the recovery stage to reduce the compressive yield stress toward zero would allow free compressive evolution in the viscoplastic dashpot and thus recover just as the viscoelastic component does. Given enough time at the elevated temperature with compressive yield stress equal to zero, the model would fully recover as is observed by Pegoretti et al. [44] in real materials.

A noteworthy point is in regard to the contributions of stress relaxation and strain recovery from the viscoelastic and viscoplastic components. Figure 4 (g) shows viscoplastic contribution to relaxation until the stress reduces below yield. In real materials it is noted that relaxation is a property of viscoelasticity (Brusselle-Dupend et al. [5, 6]). This is one reason for the importance of kinematic and isotropic hardening in a model for semicrystalline polymers, properties not accounted for in the above linear study. Such hardening rules increase the yield limit with the highest level of stress reached. In this way upon the onset of a relaxation period, the viscoplastic element stress would immediately drop below the hardened value of yield and so no relaxation contribution will take place. This is also the case during load reversal and recovery meaning only elastic forms of deformation can be recovered as is expected. The absence of strain hardening in the linear model presented here is the one discrepancy between the above

curve responses and those observed in reality however this is taken into account in the more advanced constitutive mathematics to follow.

\section{Formulation of Constitutive Mathematics}

A complete set of constitutive equations will be presented to act as a comprehensive collation of continuum mechanics constitutive theory for application to semicrystalline polymers. Perić and Dettmer [46] provide a brief outline of constitutive equations and their foundations; these will be treated in more detail here including explicit definition 
of isotropic and kinematic hardening suitable for the semicrystalline polymer case. The numerical implications of incorporating strain rate dependent viscosity expressions for a three-dimensional, large strain model will also be treated.

\subsection{Kinematics and Thermodynamics}

Let us first establish some of the kinematic and thermodynamic foundations that the theory will depend on.

Of particular importance to the measurement of finite deformation is the second order deformation gradient tensor, $\boldsymbol{F}$ [3]. A key characteristic of finite deformation is the presence of rigid body motion. A consequence of this is that some arbitrary vector within the continuum of a deforming body can be described either in terms of its position in space or relative to its initial material position. These are referred to as Eulerian and Lagrangian descriptions respectively. The deformation gradient describes the final spatial configuration of such a vector with respect to its initial material configuration. The deformation gradient is evidently associated with strain in both Lagrangian and Eulerian space, hence its importance to continuum mechanics.

Particularization of strain measurement into exclusive Lagrangian or Eulerian description results in the common measures of tensorial strain; $\boldsymbol{C}=\boldsymbol{F}^{T} \boldsymbol{F}$, the Lagrangian right Cauchy-Green strain tensor and $\boldsymbol{b}=\boldsymbol{F} \boldsymbol{F}^{T}$, the Eulerian left Cauchy-Green strain tensor. Spectral decomposition of these quantities enables the recovery of principal strain measures used widely in material science. This decomposition is treated at length by authors such as Bonet and Wood [3], Simo and Taylor [62] and Itskov [26, 27, 28] and proceeds

$$
\boldsymbol{C}=\sum_{A=1}^{3} \Lambda_{A} \boldsymbol{N}_{A} \otimes \boldsymbol{N}_{A} \quad \boldsymbol{b}=\sum_{A=1}^{3} \Lambda_{A} \boldsymbol{n}_{A} \otimes \boldsymbol{n}_{A}
$$

where $\Lambda_{A}, A=1,2,3$ are the common eigenvalues of $\boldsymbol{C}$ and $\boldsymbol{b}$ while $\boldsymbol{N}_{A}$ and $\boldsymbol{n}_{A}$ are the Lagrangian and Eulerian eigenvector triads respectively [3]. Correspondingly, $\lambda_{A}=\sqrt{\Lambda_{A}}$ are the principal stretches and $\varepsilon_{A}=$ $\ln \left(\lambda_{A}\right)$ are the logarithmic principal strains each commonly used in the experimental literature ([5,6], [20], [63] for example).

The constitutive model being treated here accounts for the parallel addition of elastic, viscoelastic and viscoplastic components (Figure 3). The constitutive behavior of the elastic element is associated with total deformation, however for the viscoelastic and viscoplastic elements it is necessary to separate total deformation into elastic and inelastic

components. Referring to Bonet and Wood [3], in tensor space this 


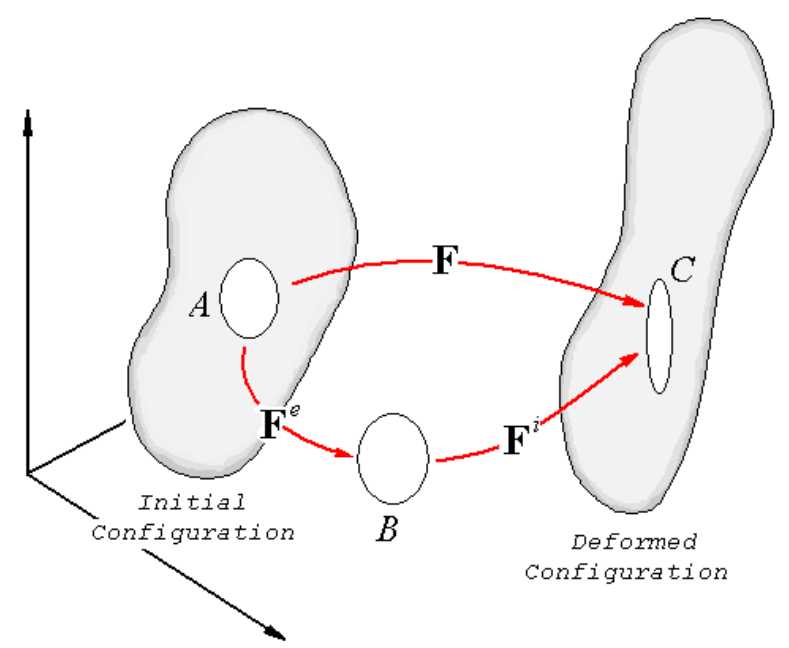

Figure 7. The multiplicative split of the deformation gradient into elastic and inelastic components.

is facilitated via the multiplicative split of the deformation gradient

$$
\boldsymbol{F}=\boldsymbol{F}^{e} \boldsymbol{F}^{i}
$$

This is diagrammatically represented in Figure 7. From the figure, where the total deformation gradient maps the initial configuration, $A$ to its deformed configuration $C$, it is conceptually equivalent to allow the elastic component $\boldsymbol{F}^{e}$ to map $A$ to some intermediate position $B$ and correspondingly for the inelastic component $\boldsymbol{F}^{i}$, to map $B$ to the final configuration $C$. From rearrangement of Equation 5.2, it is convenient to establish the elastic components of right and left Cauchy-Green strain as

$$
\boldsymbol{C}^{e}=\boldsymbol{F}^{e T} \boldsymbol{F}^{e} \quad \boldsymbol{b}^{e}=\boldsymbol{F}^{e} \boldsymbol{F}^{e T}
$$

Within continuum mechanics, a large part of the accurate expression of a materials constitutive behavior is associated with expression of the materials thermodynamic free energy, $\psi$. Referring to Reese and Govindjee [52], the expression of free energy can be given

$$
\psi=\hat{\psi}\left(\boldsymbol{C}, \xi_{1}, \xi_{2}, \ldots, \xi_{n}\right)
$$

where for some isothermal process, the free energy is functional on strain, $\boldsymbol{C}$, and some set of internal variables, $\xi_{i}, i=1,2, \ldots, n$. Note that 
because the free energy is a continuum quantity, it must be defined in terms of Lagrangian strain ${ }^{7}$.

The strain dependence of the free energy is commonly associated with elastic mechanisms [40] while the internal variables are generally associated with the inelastic response [46, 61] which gives rise to the decoupled expression treated by Perić [45]

$$
\hat{\psi}\left(\boldsymbol{C}^{e}, \xi_{1}, \xi_{2}, \ldots, \xi_{n}\right)=\hat{\mathcal{W}}\left(\boldsymbol{C}^{e}\right)+\hat{\mathcal{H}}\left(\xi_{1}, \xi_{2}, \ldots, \xi_{n}\right)
$$

where $\hat{\mathcal{W}}$ is the elastic thermodynamic potential, examples of which include the St. Venant-Kirchhoff (elastic), and Ogden (hyperelastic) models [3], while the definition of $\hat{\mathcal{H}}$ clearly depends on the nature of the internal variables. Given that $\hat{\psi}$ is associated with material deformation that can be separated into elastic and inelastic components via Equation 5.2, then $\hat{\mathcal{W}}$ is only functional on the elastic component as shown $[38,52]$. It follows that for a purely elastic material, $C^{e}=C$ such that

$$
\hat{\psi}(\boldsymbol{C})=\hat{\mathcal{W}}(\boldsymbol{C})
$$

where no internal variables are necessary.

Focusing on the particular case of an elasto-viscoelastic-viscoplastic constitutive theory, because of the parallel configuration (Figure 3), it is a common convention to define the total free energy expression via the sum of the components $[23,24,38,52]$ as

$$
\psi=\hat{\psi}_{e}(\boldsymbol{C})+\hat{\psi}_{v e}\left(\boldsymbol{C}_{v e}^{e}, \eta_{v e}\right)+\hat{\psi}_{v p}\left(\boldsymbol{C}_{v p}^{e}, \eta_{v p}, \alpha, \boldsymbol{\alpha}\right)
$$

where $\alpha$ and $\boldsymbol{\alpha}$ are viscoplastic internal variables associated with isotropic and kinematic hardening respectively, and also, contrary to the traditional form of $\hat{\psi}_{v e}$ and $\hat{\psi}_{v p}$ (for example $[52,46]$ for viscoelasticity and $[45,46,59,61]$ for viscoplasticity), the viscosity terms $\eta_{v e}$ and $\eta_{v p}$ have been included as additional internal variables. This is due to the requirement that the viscosity terms used in any constitutive model for semicrystalline polymers, have some functionality on strain (Section 3.3). We will elaborate further on this point in subsequent sections.

\subsection{StRESS}

To allow definition of the constitutive stress expression, it is necessary to observe that, following for example Holzapfel [23], Nedjar [38] and Reese and Govindjee [52], all constitutive equations must satisfy the

\footnotetext{
7 When a material is isotropic, the free energy can also be expressed as a function of Eulerian strain [52] ( $\boldsymbol{b}$ for example) however this is an unnecessary modification for the present development.
} 
$2^{\text {nd }}$ law of thermodynamics. In Lagrangian description, this can take the form of the Clausius-Plank inequality ${ }^{8}$

$$
\boldsymbol{S}: \frac{1}{2} \dot{\boldsymbol{C}}-\dot{\psi} \geq 0
$$

where $\boldsymbol{S}$ is the second Piola-Kirchhoff stress tensor. By the standard development $[38,46,52,59]$, implementation of Equation 5.7 into Equation 5.8 enables the explicit definition of the stress expression. This development is included in Appendix A. by

From Appendix A, the total second Piola-Kirchhoff stress is given

$$
\begin{aligned}
\boldsymbol{S} & =2 \frac{\partial \hat{\psi}_{e}}{\partial \boldsymbol{C}}+2 \boldsymbol{F}_{v e}^{i-1} \frac{\partial \hat{\psi}_{v e}}{\partial \boldsymbol{C}_{v e}^{e}} \boldsymbol{F}_{v e}^{i-T}+2 \boldsymbol{F}_{v p}^{i-1} \frac{\partial \hat{\psi}_{v p}}{\partial \boldsymbol{C}_{v p}^{e}} \boldsymbol{F}_{v p}^{i-T} \\
& =\boldsymbol{S}_{e}+\boldsymbol{S}_{v e}+\boldsymbol{S}_{v p}
\end{aligned}
$$

In solid mechanics, while the account of the constitutive behavior of a material is inherently Lagrangian [3], it is convenient from an implementation standpoint, to also define the Eulerian quantities. Correspondingly, the Eulerian counterpart to $\boldsymbol{S}$, is the Kirchhoff stress tensor $\boldsymbol{\tau}$. Lagrangian and Eulerian quantities are related through the concepts of push forward and pull back [3, 62] where the Kirchhoff stress can be attained from the second order push forward of $\boldsymbol{S}$ via

$$
\tau=\boldsymbol{F} \boldsymbol{S} \boldsymbol{F}^{T}
$$

Following Equation 5.10, then Equation 5.9 becomes

$$
\begin{aligned}
\boldsymbol{\tau} & =2 \boldsymbol{F} \frac{\partial \hat{\psi}_{e}}{\partial \boldsymbol{C}} \boldsymbol{F}^{T}+2 \boldsymbol{F}_{v e}^{e} \frac{\partial \hat{\psi}_{v e}}{\partial \boldsymbol{C}_{v e}^{e}} \boldsymbol{F}_{v e}^{e T}+2 \boldsymbol{F}_{v p}^{e} \frac{\partial \hat{\psi}_{v p}}{\partial \boldsymbol{C}_{v p}^{e}} \boldsymbol{F}_{v p}^{e T} \\
& =\boldsymbol{\tau}_{e}+\boldsymbol{\tau}_{v e}+\boldsymbol{\tau}_{v p}
\end{aligned}
$$

Because of the functionality of $\boldsymbol{\tau}_{v e}$ and $\boldsymbol{\tau}_{v p}$ on the elastic component of strain, it remains to define the evolution of inelastic strain ${ }^{9}$.

\subsection{Viscoelastic eVolution equations}

Limiting our attention to the evolution of the viscoelastic response, following from Appendix A, this must satisfy the inequality

$$
\boldsymbol{\tau}_{v e}:\left(-\frac{1}{2}\left(\mathcal{L}_{v} \boldsymbol{b}_{v e}^{e}\right) \boldsymbol{b}_{v e}^{e-1}\right)-\frac{\partial \hat{\psi}_{v e}}{\partial \eta_{v e}} \cdot \dot{\eta}_{v e} \geq 0
$$

\footnotetext{
8 also commonly referred to as the Clausius-Duhem inequality.

${ }^{9}$ Note $\boldsymbol{C}^{e}=\boldsymbol{F}^{i-T} \boldsymbol{C} \boldsymbol{F}^{i-1}$ such that, given the inelastic evolution, $\boldsymbol{C}^{e}$ can be determined
} 
The $-\frac{\partial \hat{\psi}_{v e}}{\partial \eta_{v e}} \cdot \dot{\eta}_{v e}$ term is associated with the evolution of the viscosity internal variable and will be treated subsequently. The remaining term is associated with strain evolution and is of primary interest.

Reese and Govindjee [52] introduce the expression

$$
-\frac{1}{2}\left(\mathcal{L}_{v} \boldsymbol{b}_{v e}^{e}\right) \boldsymbol{b}_{v e}^{e-1}:=\mathcal{V}^{-1}: \boldsymbol{\tau}_{v e}
$$

where now the left hand side of Equation 5.12 becomes

$$
\tau: \mathcal{V}^{-1}: \tau \geq 0
$$

which is satisfied for all cases of $\boldsymbol{\tau}$ providing $\mathcal{V}^{-1}$ is positive definite.

The left hand term of Equation 5.13 is related to the rate of inelastic material strain through operator split theory. For the current investigation it is sufficient to draw the analogy between Equation 5.13 and Equation $4.2_{2}$ of the infinitesimal 1D theory, where here $\mathcal{V}^{-1}$ represents the inverse viscosity in fourth order tensor space.

An inverse viscosity term suitable for polymers has been proposed by Reese and Govindjee [52] as

$$
\mathcal{V}^{-1}=\frac{1}{2 \eta_{D}}\left(\boldsymbol{I}_{4}^{\prime}-\frac{1}{3} \boldsymbol{I} \otimes \boldsymbol{I}\right)+\frac{1}{9 \eta_{V}} \boldsymbol{I} \otimes \boldsymbol{I}
$$

where $\eta_{D}$ and $\eta_{V}$ are deviatoric and volumetric components of viscosity respectively. The authors indicate that the viscosity terms $\eta_{D}$ and $\eta_{V}$ could be strain dependent but offer no further development. The terms $\left(\boldsymbol{I}_{4}^{\prime}-\frac{1}{3} \boldsymbol{I} \otimes \boldsymbol{I}\right)$ and $\boldsymbol{I} \otimes \boldsymbol{I}$ operate through the double contraction with $\boldsymbol{\tau}_{v e}$ to isolate the deviatoric and volumetric components of stress respectively (Simo [59]). Here $I_{4 i j k l}^{\prime}=\frac{1}{2}\left(\delta_{i k} \delta_{j l}+\delta_{i l} \delta_{j k}\right)$ is the supersymmetric 4th order identity tensor and $I_{i j}=\delta_{i j}$ is the 2nd order identity tensor (Bonet and Wood [3]). Holzapfel [23] notes that based on experimental results, the inelastic evolution of most polymeric materials is wholly deviatoric and consequently, the volumetric term from (5.15) can be ignored (i.e. $\eta_{v e}=\eta_{D}$ ). This property is further confirmed in the works of Perić and Dettmer [46] and Reese and Govindjee [52].

It is left to establish the actual form of viscosity expression that would allow adequate account of the strain rate dependence observed for semicrystalline polymers.

In the context of one-dimensional, finite deformation, Brusselle-Dupend et al. $[5,6]$ have proposed a viscosity expression for the viscoelastic response of semicrystalline polypropylene as

$$
\eta_{v e}=\frac{h_{0}-h_{1} \exp \left\{h_{2} \frac{\left|\dot{\varepsilon}^{e}\right|+3 \times 10^{-5}}{\left|\dot{\varepsilon}^{i}\right|}\right\}-h_{3} \exp \left\{h_{4} \frac{\left|\dot{\varepsilon}^{e}\right|+3 \times 10^{-5}}{\left|\dot{\varepsilon}^{i}\right|}\right\}}{\left(\left|\dot{\varepsilon}^{e}\right|+3 \times 10^{-5}\right)^{n e}}
$$


where $h_{0,1, \ldots, 4}$ and ne are material constants. This equation was developed due to the reported inadequacies with the conventionally used Eyring equation $[16,29]$ when applied to semicrystalline polymer response.

For three-dimensionality, the viscosity of a material remains a scalar quantity (as per Equation 5.15) however the multidimensional dependencies of this value have not been treated with any generality in the literature, particularly for the case at hand. As a consequence, it would appear that a significant amount of experimental work is needed to allow development of a three-dimensional analog to Equation $5.16^{10}$.

Developing on this concept briefly, because of its strain dependence, the viscosity is considered to be an internal variable in a similar way to the strain hardening terms from plasticity. Following from Perić [48], Reese and Govindjee [52] and Simo and Hughes [61] it is most desirable to define the constitutive behavior of internal variables via their evolution, i.e.

$$
\dot{\eta}_{v e}=\hat{\eta}_{v e}\left(\boldsymbol{C}_{v e}^{i}, \eta_{v e}\right)=\hat{\eta}_{v e}\left(\boldsymbol{F}^{T} \boldsymbol{b}_{v e}^{e-1} \boldsymbol{F}, \eta_{v e}\right)
$$

By specifying the constitutive behavior of the viscosity in this way, a numerical solution scheme such as Closest point projection, conventionally used in plasticity $[45,61]$, can be used for the simultaneous solution of strain (Equation 5.13) and viscosity (Equation 5.17). For the case of linear viscosity the viscoelastic constitutive theory can be implemented via a local Newton iteration scheme [52], a method like closest point projection can be interpreted as performing this Newton iteration for two or more mutually dependent variables.

An additional requirement of the constitutive behavior of the viscosity is that the evolution expression Equation 5.17, and the free energy component $\hat{\mathcal{H}}_{v e}\left(\eta_{v e}\right)$, must suitably satisfy the right hand side of the dissipation requirement, Equation 5.12.

\subsection{Viscoplastic EVOLUtion EQUATIONS}

The derivation of the constitutive equations for one-dimensional viscoplasticity with isotropic and kinematic hardening is presented for reference in Appendix B.

For three-dimensional large strain, as was the case for viscoelasticity, the viscoplastic constitutive equations must satisfy the thermodynamic

\footnotetext{
10 From a purely theoretical stand point, it would be equivalent to substitute principal vector quantities (developed from Equation 5.1) for the scalar strain rates in Equation 5.1 however this is only a means of conceptual implementation.
} 
dissipation requirement (Appendix A)

$$
\boldsymbol{\tau}_{v p}:\left(-\frac{1}{2}\left(\mathcal{L}_{v} \boldsymbol{b}_{v p}^{e}\right) \boldsymbol{b}_{v p}^{e-1}\right)-\frac{\partial \hat{\psi}_{v p}}{\partial \eta_{v p}} \cdot \dot{\eta}_{v p}-\frac{\partial \hat{\psi}_{v p}}{\partial \alpha} \cdot \dot{\alpha}-\frac{\partial \hat{\psi}_{v p}}{\partial \boldsymbol{\alpha}}: \dot{\boldsymbol{\alpha}} \geq 0
$$

where again viscosity is implemented as a strain dependent internal variable and the scalar $\alpha$ and tensorial $\boldsymbol{\alpha}$ internal variables are associated with isotropic and kinematic hardening mechanisms respectively (akin to $\alpha_{i s o}$ and $\alpha_{k i n}$ from the one-dimensional theory).

It is again pertinent to introduce the stress space conjugates to the isotropic and kinematic hardening internal variables, namely $q$ and $\boldsymbol{q}$. By a similar argument to that which motivated the formulation of Equation 5.5, referring to Perić [45], Simo [59] and Simo and Hughes [61], the viscoplastic free energy can be expressed

$$
\hat{\psi}\left(\boldsymbol{C}_{v p}^{e}, \eta_{v p}, \alpha, \boldsymbol{\alpha}\right)=\hat{\mathcal{W}}\left(\boldsymbol{C}_{v p}^{e}\right)+\hat{\mathcal{H}}_{v p}^{\eta}\left(\eta_{v p}\right)+\hat{\mathcal{H}}_{v p}^{\alpha}(\alpha)+\hat{\mathcal{H}}_{v p}^{\boldsymbol{\alpha}}(\boldsymbol{\alpha})
$$

Implementing a combined, linear isotropic/kinematic hardening law following Hughes [25] and Simo and Hughes [61], it can be shown that

$$
\begin{aligned}
\hat{\mathcal{H}}_{v p}^{\alpha} & =\frac{1}{2} \theta \overline{\mathrm{H}} \alpha^{2} & \hat{\mathcal{H}}_{v p}^{\boldsymbol{\alpha}} & =\frac{1}{2}(1-\theta) \overline{\mathrm{H}} \boldsymbol{\alpha}: \boldsymbol{\alpha} \\
\frac{\partial \hat{\mathcal{H}}_{v p}^{\alpha}}{\partial \alpha} & =\theta \overline{\mathrm{H}} \alpha=-q & \frac{\partial \hat{\mathcal{H}}_{v p}^{\boldsymbol{\alpha}}}{\partial \boldsymbol{\alpha}} & =(1-\theta) \overline{\mathrm{H}} \boldsymbol{\alpha}=-\boldsymbol{q}
\end{aligned}
$$

where $\overline{\mathrm{H}}$ is a constant parameter representing the combined hardening modulus and $\theta$ defines the proportions of isotropic and kinematic hardening; $\theta \in[0,1]$. It follows directly from Equation 5.21 that the actual hardening moduli are given

$$
-\frac{\partial^{2} \hat{\mathcal{H}}_{v p}^{\alpha}}{\partial \alpha^{2}}=-\theta \overline{\mathrm{H}}=\frac{\partial q}{\partial \alpha} \quad-\frac{\partial^{2} \hat{\mathcal{H}}_{v p}^{\boldsymbol{\alpha}}}{\partial \boldsymbol{\alpha}^{2}}=-(1-\theta) \overline{\mathrm{H}} \boldsymbol{I} \otimes \boldsymbol{I}=\frac{\partial \boldsymbol{q}}{\partial \boldsymbol{\alpha}}
$$

These constant terms are inferred to be suitable for the current purpose however for more complex hardening characteristics, alternate specification of $\hat{\mathcal{H}}_{v p}^{\alpha}$ and $\hat{\mathcal{H}}_{v p}^{\alpha}$ can be used.

Given the stress space hardening internal variables, it is possible to carry out a three-dimensional study of the effects of strain hardening on the yield surface as was done for the one-dimensional case (Appendix B). Following Simo [59], Simo and Hughes [61] and Zienkiewicz and Taylor [66], selection of the von-Mises yield surface gives the yield function

$$
f\left(\boldsymbol{\tau}_{v p}+\boldsymbol{q}, q\right)=\left\|\boldsymbol{\tau}_{v p}+\boldsymbol{q}\right\|-\sqrt{\frac{2}{3}}\left(\sigma_{Y}-q\right)
$$

Physically $\sqrt{\frac{2}{3}}\left(\sigma_{Y}-q\right)$ quantifies the radius of the yield surface, while $-\boldsymbol{q}$ defines its center. As for the viscoelastic case, here we will assume 
inelasticity to have only deviatoric components such that now

$$
f\left(\overline{\boldsymbol{\tau}}_{v p}+\boldsymbol{q}, q\right)=\left\|\overline{\boldsymbol{\tau}}_{v p}+\boldsymbol{q}\right\|-\sqrt{\frac{2}{3}}\left(\sigma_{Y}-q\right)
$$

where $\overline{\boldsymbol{\tau}}_{v p}=\operatorname{dev}\left[\boldsymbol{\tau}_{v p}\right]$ and now the internal variable $\boldsymbol{q}$ is wholly deviatoric such that $\operatorname{tr}[\boldsymbol{q}]=0$.

Following a similar development as for the one-dimensional case in Appendix B, Perzyna [49] and subsequent authors such as Simo [59], Simo and Hughes [61], Perić and Owen [47], Perić and Dettmer [46] and Rosati and Valoroso [53] define the evolution expression

$$
-\frac{1}{2}\left(\mathcal{L}_{v} \boldsymbol{b}_{v p}^{e}\right) \boldsymbol{b}_{v p}^{e-1}=\frac{\left\langle f\left(\overline{\boldsymbol{\tau}}_{v p}+\boldsymbol{q}, q\right)\right\rangle}{2 \eta_{v p}} \frac{\partial f\left(\overline{\boldsymbol{\tau}}_{v p}+\boldsymbol{q}, q\right)}{\partial \overline{\boldsymbol{\tau}}_{v p}}
$$

Once again, an analogy is evident between this inelastic evolution and that reported for the one-dimensional case in Appendix B.

Referring to the explicit formulations of Simo [59], Simo and Hughes [61] and Zienkiewicz and Taylor [66], the evolution of the strain space hardening internal variables in tensor space are subsequently defined

$$
\begin{aligned}
& \dot{\alpha}=\frac{\left\langle f\left(\overline{\boldsymbol{\tau}}_{v p}+\boldsymbol{q}, q\right)\right\rangle}{2 \eta_{v p}} \frac{\partial f\left(\overline{\boldsymbol{\tau}}_{v p}+\boldsymbol{q}, q\right)}{\partial q} \\
& \dot{\boldsymbol{\alpha}}=\frac{\left\langle f\left(\overline{\boldsymbol{\tau}}_{v p}+\boldsymbol{q}, q\right)\right\rangle}{2 \eta_{v p}} \frac{\partial f\left(\overline{\boldsymbol{\tau}}_{v p}+\boldsymbol{q}, q\right)}{\partial \boldsymbol{q}}
\end{aligned}
$$

Returning our attention to the dissipation requirement, Equation 5.18, neglecting the viscosity term ${ }^{11}$, substitution of Equations 5.21, $5.24,5.25,5.26$ and 5.27 yields with some manipulation

$$
\left(\boldsymbol{\tau}_{v p}+\boldsymbol{q}\right): \frac{\langle f\rangle}{2 \eta_{v p}} \frac{\left(\boldsymbol{I}_{4}^{\prime}-\frac{1}{3} \boldsymbol{I} \otimes \boldsymbol{I}\right)}{\left\|\boldsymbol{\tau}_{v p}+\boldsymbol{q}\right\|}:\left(\boldsymbol{\tau}_{v p}+\boldsymbol{q}\right)+q \cdot \sqrt{\frac{2}{3}} \frac{\langle f\rangle}{2 \eta_{v p}} \geq 0
$$

which is unconditionally satisfied given that from definition of the ramp function (Equation B.8), $\langle\cdot\rangle \geq 0$ and also from the known behavior of isotropic hardening $q \geq 0$.

The considerations regarding strain rate dependence of the viscosity for the viscoplastic case are identical in form to those presented in Section 5.3 for the viscoelastic case. Consequently, no further discussion is necessary within the current investigation.

Exact specification of the elastic thermodynamic potential terms, $\hat{\mathcal{W}}_{e}(\boldsymbol{C}), \hat{\mathcal{W}}_{v e}\left(\boldsymbol{C}_{v e}^{e}\right)$ and $\hat{\mathcal{W}}_{v e}\left(\boldsymbol{C}_{v p}^{e}\right)$, to be used within a constitutive theory for semicrystalline polymers is largely case specific. Generally any

\footnotetext{
11 Satisfaction of the dissipation requirement must be considered during formulation of an expression for viscoplastic viscosity, as was the case for viscoelasticity discussed in Section 5.3.
} 
elastic or hyperelastic form of thermodynamic potential could be chosen (see Bonet and Wood [3]). A suggestion would be to use hyperelastic Ogden potentials for the elastic (Simo and Taylor [62]) and viscoelastic (Reese and Govindjee [52]) elements and an elastic type St. VenantKirchhoff potential for the viscoplastic element (Simo [59]). The choice of free energy expression must be governed by experimental observation because it must represent the thermodynamic potential of the material. As such final selection of the exact free energy form requires response curves from physical tests. For a more detailed discussion of hyperelastic free energy expressions for numerical use see Simo and Taylor [62], Simo [59], Perić and Owen [47], Reese and Govindjee [52], Simo and Hughes [61] and Perić and Dettmer [46].

\section{Conclusions}

The aim of this article was to determine the most appropriate constitutive theory for semicrystalline polymers based on a review of the literature. From both a microscopic and macroscopic perspective, deformation characteristics such as elasto-viscoelasto-viscoplasticity, yield point, isotropic and kinematic hardening and strain dependent viscosities have all been shown to be of significant importance. A model has been proposed that accounts for all these complex characteristics.

A preliminary study has shown the parallel rheological configuration of the chosen model to be suitable across a wide range of complex deformation behaviors based on comparison with experimental observations from the literature. Specific validation of the selected nonlinear, continuum mechanics constitutive theory is largely dependent on the explicit generation of material specific parameters (to be published at a later date), however the accuracy of such theories for the representation of similar materials (rubbers, elastomers etc) has been well documented.

The model's constitutive mathematics are presented in their most general form with the objective of being broadly applicable to all semicrystalline polymers.

\section{Acknowledgements}

The authors would like to acknowledge Gough Plastic Pty Ltd for financial support towards this research. 


\section{Appendix}

\section{Appendix A. Derivation of the dissipation requirement}

For a constant temperature process, the Clausius-Plank form of the 2nd law of thermodynamics is expressed in Lagrangian form via

$$
\boldsymbol{S}: \frac{1}{2} \dot{\boldsymbol{C}}-\dot{\psi} \geq 0
$$

where $\dot{C}$ is the time differential of the right Cauchy-Green strain tensor, : denotes the tensor double contraction (see Bonet and Wood [3]) and $\dot{\psi}$ is the time derivative of the free energy expression. The free energy expression $\psi$ is expressed in terms of elastic, viscoelastic and viscoplastic components as

$$
\psi=\hat{\psi}_{e}(\boldsymbol{C})+\hat{\psi}_{v e}\left(\boldsymbol{C}_{v e}^{e}, \eta_{v e}\right)+\hat{\psi}_{v p}\left(\boldsymbol{C}_{v p}^{e}, \eta_{v p}, \alpha, \boldsymbol{\alpha}\right)
$$

Note here that for the semicrystalline polymer application, the viscosity terms $\eta_{v e}$ and $\eta_{v p}$ have become internal variables because of their functionality on strain (rate). Recalling the definition $\boldsymbol{C}=\boldsymbol{F}^{T} \boldsymbol{F}$ and the multiplicative split of the deformation gradient Equation 5.3, it is convenient to substitute the relationships

$$
\boldsymbol{C}_{v e}^{e}=\boldsymbol{F}_{v e}^{i-T} \boldsymbol{C F}_{v e}^{i-1} \text { and } \boldsymbol{C}_{v p}^{e}=\boldsymbol{F}_{v p}^{i-T} \boldsymbol{C F}_{v p}^{i-1}
$$

such that now

$$
\psi=\hat{\psi}_{e}(\boldsymbol{C})+\hat{\psi}_{v e}\left(\boldsymbol{F}_{v e}^{i-T} \boldsymbol{C} \boldsymbol{F}_{v e}^{i-1}, \eta_{v e}\right)+\hat{\psi}_{v p}\left(\boldsymbol{F}_{v p}^{i-T} \boldsymbol{C} \boldsymbol{F}_{v p}^{i-1}, \eta_{v p}, \alpha, \boldsymbol{\alpha}\right)
$$

Following Reese and Govindjee [52] and Nedjar [38], taking the chain rule differential of (A.4) with respect to time gives

$$
\begin{aligned}
\dot{\psi} & =\frac{\partial \hat{\psi}_{e}}{\partial \boldsymbol{C}}: \dot{\boldsymbol{C}}+\frac{\partial \hat{\psi}_{v e}}{\partial \boldsymbol{C}_{v e}^{e}}: \frac{\partial \boldsymbol{C}_{v e}^{e}}{\partial \boldsymbol{C}}: \dot{\boldsymbol{C}}+\frac{\partial \hat{\psi}_{v e}}{\partial \boldsymbol{C}_{v e}^{e}}: \frac{\partial \boldsymbol{C}_{v e}^{e}}{\partial \boldsymbol{F}_{v e}^{i}}: \dot{\boldsymbol{F}}_{v e}^{i} \\
& +\frac{\partial \hat{\psi}_{v p}}{\partial \boldsymbol{C}_{v p}^{e}}: \frac{\partial \boldsymbol{C}_{v p}^{e}}{\partial \boldsymbol{C}}: \dot{\boldsymbol{C}}+\frac{\partial \hat{\psi}_{v p}}{\partial \boldsymbol{C}_{v p}^{e}}: \frac{\partial \boldsymbol{C}_{v p}^{e}}{\partial \boldsymbol{F}_{v p}^{i}}: \dot{\boldsymbol{F}}_{v p}^{i} \\
& +\frac{\partial \hat{\psi}_{v e}}{\partial \eta_{v e}} \cdot \dot{\eta}_{v e}+\frac{\partial \hat{\psi}_{v p}}{\partial \eta_{v p}} \cdot \dot{\eta}_{v p}+\frac{\partial \hat{\psi}_{v p}}{\partial \alpha} \cdot \dot{\alpha}+\frac{\partial \hat{\psi}_{v p}}{\partial \boldsymbol{\alpha}}: \dot{\boldsymbol{\alpha}}
\end{aligned}
$$

Separately evaluating specific terms from within (A.5), for the $\dot{\boldsymbol{C}}$ terms, where the subscript $n=v e, v p$

$$
\begin{aligned}
\frac{\partial \hat{\psi}_{n}}{\partial \boldsymbol{C}_{n}^{e}}: \frac{\partial \boldsymbol{C}_{n}^{e}}{\partial \boldsymbol{C}}: \dot{\boldsymbol{C}} & =\frac{\partial \hat{\psi}_{n}}{\partial \boldsymbol{C}_{n}^{e}}: \frac{\partial\left(\boldsymbol{F}_{n}^{i-T} \boldsymbol{C F}_{n}^{i-1}\right)}{\partial \boldsymbol{C}}: \dot{\boldsymbol{C}} \\
& =\frac{\partial \hat{\psi}_{n}}{\partial \boldsymbol{C}_{n}^{e}}: \boldsymbol{F}_{n}^{i-T} \boldsymbol{I}_{4} \boldsymbol{F}_{n}^{i-1}: \dot{\boldsymbol{C}}
\end{aligned}
$$


where from Itskov [26, 28] and Kintzel [30], the derivative of some second order tensor $A_{i j}$ with respect to its self is given by $\partial A_{i j} / \partial A_{k l}=$ $I_{4 i j k l}=\delta_{i j} \delta_{k l}$, the fourth order identity tensor. Also, a useful identity used by Reese and Govindjee [52] associated with the double tensor contraction is $\boldsymbol{A}: \boldsymbol{B}=\boldsymbol{C}^{-1} \boldsymbol{A} \boldsymbol{D}^{-1}: \boldsymbol{D} \boldsymbol{B} \boldsymbol{C}$. Correspondingly

$$
=2 \boldsymbol{F}_{n}^{i-1} \frac{\partial \hat{\psi}_{n}}{\partial \boldsymbol{C}_{n}^{e}} \boldsymbol{F}_{n}^{i-T}: \frac{1}{2} \dot{\boldsymbol{C}}
$$

For the $\dot{\boldsymbol{F}}_{n}^{i}$ terms

$$
\begin{aligned}
\frac{\partial \hat{\psi}_{n}}{\partial \boldsymbol{C}_{n}^{e}}: \frac{\partial \boldsymbol{C}_{n}^{e}}{\partial \boldsymbol{F}_{n}^{i}}: \dot{\boldsymbol{F}}_{n}^{i} & =\frac{\partial \hat{\psi}_{n}}{\partial \boldsymbol{C}_{n}^{e}}: \frac{\partial\left(\boldsymbol{F}_{n}^{i-T} \boldsymbol{C F}_{n}^{i-1}\right)}{\partial \boldsymbol{F}_{n}^{i}}: \dot{\boldsymbol{F}}_{n}^{i} \\
& =\frac{\partial \hat{\psi}_{n}}{\partial \boldsymbol{C}_{n}^{e}}:\left(\frac{\partial \boldsymbol{F}_{n}^{i-T}}{\partial \boldsymbol{F}_{n}^{i T}} \boldsymbol{C} \boldsymbol{F}_{n}^{i-1}: \dot{\boldsymbol{F}}_{n}^{i T}+\partial \boldsymbol{F}_{n}^{i-T} \boldsymbol{C} \frac{\partial \boldsymbol{F}_{n}^{i-1}}{\partial \boldsymbol{F}_{n}^{i}}: \dot{\boldsymbol{F}}_{n}^{i}\right)
\end{aligned}
$$

From Itskov [26, 28], for second order tensors $\partial \boldsymbol{A}^{-1} / \partial \boldsymbol{A}=-\boldsymbol{A}^{-1} \otimes \boldsymbol{A}^{-1}$ and also $\boldsymbol{A} \otimes \boldsymbol{B}: \boldsymbol{C}=\boldsymbol{A} \boldsymbol{C B}$. Using these properties and the double contraction identity used in (A.6) then with some development

$$
=\boldsymbol{F}_{n}^{i-1} \frac{\partial \hat{\psi}_{n}}{\partial \boldsymbol{C}_{n}^{e}} \boldsymbol{F}_{n}^{i-T}:\left(-\dot{\boldsymbol{F}}_{n}^{i T} \boldsymbol{F}_{n}^{i-T} \boldsymbol{C}-\boldsymbol{C F}_{n}^{i-1} \dot{\boldsymbol{F}}_{n}^{i}\right)
$$

Using (A.6) and (A.7) in (A.5) and substituting this for the free energy time differential term in (A.1) gives the new form of the ClausiusPlank inequality for the semicrystalline polymers as

$$
\begin{aligned}
& \left(\boldsymbol{S}-2 \frac{\partial \hat{\psi}_{e}}{\partial \boldsymbol{C}}-2 \boldsymbol{F}_{v e}^{i-1} \frac{\partial \hat{\psi}_{v e}}{\partial \boldsymbol{C}_{v e}^{e}} \boldsymbol{F}_{v e}^{i-T}-2 \boldsymbol{F}_{v p}^{i-1} \frac{\partial \hat{\psi}_{v p}}{\partial \boldsymbol{C}_{v p}^{e}} \boldsymbol{F}_{v p}^{i-T}\right): \frac{1}{2} \dot{\boldsymbol{C}} \\
& +\boldsymbol{F}_{v e}^{i-1} \frac{\partial \hat{\psi}_{v e}}{\partial \boldsymbol{C}_{v e}^{e}} \boldsymbol{F}_{v e}^{i-T}:\left(\dot{\boldsymbol{F}}_{v e}^{i T} \boldsymbol{F}_{v e}^{i-T} \boldsymbol{C}+\boldsymbol{C F}_{v e}^{i-1} \dot{\boldsymbol{F}}_{v e}^{i}\right) \\
& +\boldsymbol{F}_{v p}^{i-1} \frac{\partial \hat{\psi}_{v p}}{\partial \boldsymbol{C}_{v p}^{e}} \boldsymbol{F}_{v p}^{i-T}:\left(\dot{\boldsymbol{F}}_{v p}^{i T} \boldsymbol{F}_{v p}^{i-T} \boldsymbol{C}+\boldsymbol{C} \boldsymbol{F}_{v p}^{i-1} \dot{\boldsymbol{F}}_{v p}^{i}\right) \\
& -\frac{\partial \hat{\psi}_{v e}}{\partial \eta_{v e}} \cdot \dot{\eta}_{v e}-\frac{\partial \hat{\psi}_{v p}}{\partial \eta_{v p}} \cdot \dot{\eta}_{v p}-\frac{\partial \hat{\psi}_{v p}}{\partial \alpha} \cdot \dot{\alpha}-\frac{\partial \hat{\psi}_{v p}}{\partial \boldsymbol{\alpha}}: \dot{\boldsymbol{\alpha}} \geq 0
\end{aligned}
$$

By the standard argument treated by authors such as Simo [59], Reese and Govindjee [52], Perić and Owen [48], Nedjar [38, 39] and Perić and Dettmer [46], the first term of (A.16) is equated to zero such 
that

$$
\begin{aligned}
\boldsymbol{S} & =2 \frac{\partial \hat{\psi}_{e}}{\partial \boldsymbol{C}}+2 \boldsymbol{F}_{v e}^{i-1} \frac{\partial \hat{\psi}_{v e}}{\partial \boldsymbol{C}_{v e}^{e}} \boldsymbol{F}_{v e}^{i-T}+2 \boldsymbol{F}_{v p}^{i-1} \frac{\partial \hat{\psi}_{v p}}{\partial \boldsymbol{C}_{v p}^{e}} \boldsymbol{F}_{v p}^{i-T} \\
& =\boldsymbol{S}_{e}+\boldsymbol{S}_{v e}+\boldsymbol{S}_{v p}
\end{aligned}
$$

Also, the Eulerian counterpart, the Kirchhoff stress tensor, can be attained via the second order push forward of (A.9) [3,62] such that

$$
\begin{aligned}
\boldsymbol{\tau} & =2 \boldsymbol{F} \frac{\partial \hat{\psi}_{e}}{\partial \boldsymbol{C}} \boldsymbol{F}^{T}+2 \boldsymbol{F}_{v e}^{e} \frac{\partial \hat{\psi}_{v e}}{\partial \boldsymbol{C}_{v e}^{e}} \boldsymbol{F}_{v e}^{e T}+2 \boldsymbol{F}_{v p}^{e} \frac{\partial \hat{\psi}_{v p}}{\partial \boldsymbol{C}_{v p}^{e}} \boldsymbol{F}_{v p}^{e T} \\
& =\boldsymbol{\tau}_{e}+\boldsymbol{\tau}_{v e}+\boldsymbol{\tau}_{v p}
\end{aligned}
$$

Using (A.10) it is now possible to further develop (A.7). Firstly exploiting the symmetric nature of $\boldsymbol{C}$, then

$$
\frac{\partial \hat{\psi}_{n}}{\partial \boldsymbol{C}_{n}^{e}}: \frac{\partial \boldsymbol{C}_{n}^{e}}{\partial \boldsymbol{F}_{n}^{i}}: \dot{\boldsymbol{F}}_{n}^{i}=2 \boldsymbol{F}_{n}^{i-1} \frac{\partial \hat{\psi}_{n}}{\partial \boldsymbol{C}_{n}^{e}} \boldsymbol{F}_{n}^{i-T}:-\boldsymbol{C F}_{n}^{i-1} \dot{\boldsymbol{F}}_{n}^{i}
$$

Using the previously noted double contraction identity

$$
=-2 \boldsymbol{F}_{n}^{e} \frac{\partial \hat{\psi}_{n}}{\partial \boldsymbol{C}_{n}^{e}} \boldsymbol{F}_{n}^{e T}: \boldsymbol{F}^{-T} \boldsymbol{C} \boldsymbol{F}_{n}^{i-1} \dot{\boldsymbol{F}}_{n}^{i} \boldsymbol{F}^{-1}
$$

Substituting the result from (A.10) and exploiting the multiplicative split of the deformation gradient, Equation 5.3, then with some development

$$
=-\boldsymbol{\tau}_{n}: \boldsymbol{F}_{n}^{e} \dot{\boldsymbol{F}}_{n}^{i} \boldsymbol{F}_{n}^{i-1} \boldsymbol{F}_{n}^{e-1}
$$

It is convenient here to introduce the Lie derivative of the elastic left Cauchy-Green strain tensor, $\mathcal{L}_{v} \boldsymbol{b}_{n}^{e}$ where from Bonet and Wood [3], Perić and Owen [48], Simo and Hughes [61], Itskov [26], Perić and Dettmer [46] and Kintzel [30]

$$
\mathcal{L}_{v} \boldsymbol{b}_{n}^{e}=\boldsymbol{F} \overline{\left(\boldsymbol{F}^{-1} \boldsymbol{b}_{n}^{e} \boldsymbol{F}^{-T}\right)} \boldsymbol{F}^{T}=\dot{\boldsymbol{b}}_{n}^{e}-\boldsymbol{l} \boldsymbol{b}_{n}^{e}-\boldsymbol{b}_{n}^{e} \boldsymbol{l}^{T}
$$

where $\boldsymbol{l}$ is the spatial velocity gradient given by $\boldsymbol{l}=\dot{\boldsymbol{F}} \boldsymbol{F}^{-1}[52,46]$. Making further use of Equation 5.3, and noting that $\boldsymbol{b}_{n}^{e}=\boldsymbol{F}_{n}^{e} \boldsymbol{F}_{n}^{e T}$ then after some development

$$
\mathcal{L}_{v} \boldsymbol{b}_{n}^{e}=-\boldsymbol{F}_{n}^{e} \dot{\boldsymbol{F}}_{n}^{i} \boldsymbol{F}_{n}^{i-1} \boldsymbol{F}_{n}^{e-1} \boldsymbol{b}_{n}^{e}-\boldsymbol{b}_{n}^{e} \boldsymbol{F}_{n}^{e-T} \boldsymbol{F}_{n}^{i-T} \dot{\boldsymbol{F}}_{n}^{i T} \boldsymbol{F}_{n}^{e T}
$$

Observing the symmetry of $\boldsymbol{b}_{n}^{e}$, rearranging gives

$$
\boldsymbol{F}_{n}^{e} \dot{\boldsymbol{F}}_{n}^{i} \boldsymbol{F}_{n}^{i-1} \boldsymbol{F}_{n}^{e-1}=-\frac{1}{2}\left(\mathcal{L}_{v} \boldsymbol{b}_{n}^{e}\right) \boldsymbol{b}_{n}^{e-1}
$$


such that now (A.11) becomes

$$
\frac{\partial \hat{\psi}_{n}}{\partial \boldsymbol{C}_{n}^{e}}: \frac{\partial \boldsymbol{C}_{n}^{e}}{\partial \boldsymbol{F}_{n}^{i}}: \dot{\boldsymbol{F}}_{n}^{i}=\boldsymbol{\tau}_{n}: \frac{1}{2}\left(\mathcal{L}_{v} \boldsymbol{b}_{n}^{e}\right) \boldsymbol{b}_{n}^{e-1}
$$

In view of (A.9) and (A.15) it is a direct development to show that the Clausius-Plank inequality, (A.16), takes the final form

$$
\begin{gathered}
\boldsymbol{\tau}_{v e}:\left(-\frac{1}{2}\left(\mathcal{L}_{v} \boldsymbol{b}_{v e}^{e}\right) \boldsymbol{b}_{v e}^{e-1}\right)+\boldsymbol{\tau}_{v p}:\left(-\frac{1}{2}\left(\mathcal{L}_{v} \boldsymbol{b}_{v p}^{e}\right) \boldsymbol{b}_{v p}^{e-1}\right) \\
-\frac{\partial \hat{\psi}_{v e}}{\partial \eta_{v e}} \cdot \dot{\eta}_{v e}-\frac{\partial \hat{\psi}_{v p}}{\partial \eta_{v p}} \cdot \dot{\eta}_{v p}-\frac{\partial \hat{\psi}_{v p}}{\partial \alpha} \cdot \dot{\alpha}-\frac{\partial \hat{\psi}_{v p}}{\partial \boldsymbol{\alpha}}: \dot{\boldsymbol{\alpha}} \geq 0
\end{gathered}
$$

\section{Appendix B. Viscoplastic constitutive equations in 1D}

The one dimensional viscoplastic rheological element discussed here is comparable to that treated by Simo and Hughes [61], Owen and Hinton [43] and Zienkiewicz and Taylor [66]. The 1D rheological form is provided in Figure B.8.

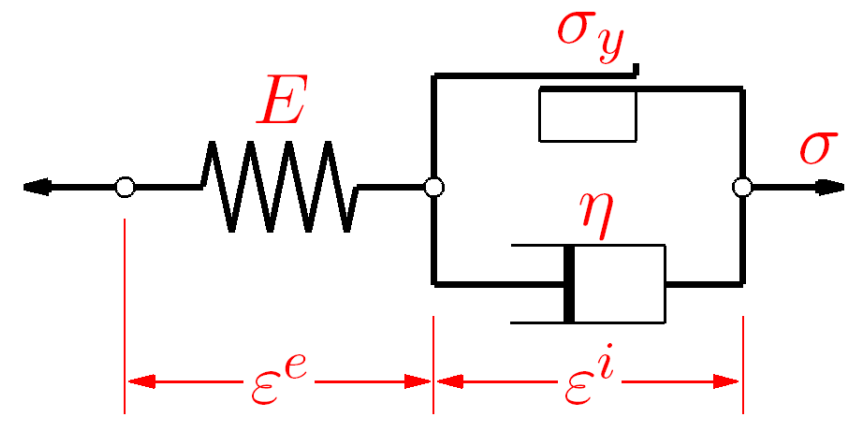

Figure B.8. One-dimensional elasto-viscoplastic rheological element (based on figure supplied by Simo and Hughes [61]).

From the figure it can be observed that the total element strain is the sum of the elastic and inelastic components

$$
\varepsilon=\varepsilon^{e}+\varepsilon^{i}
$$

The spring element accounts for the instantaneous elastic behavior where total element stress is related to elastic strain via Hooke's law

$$
\sigma=E \varepsilon^{e}=E\left(\varepsilon-\varepsilon^{i}\right)
$$

Within the inelastic element, firstly from time independent plasticity $[43,61]$, the friction slider is known to be only capable of carrying stress 
levels up to and including its yield threshold $\left(\sigma_{y}\right.$ in the case of perfect plasticity). Inelastic evolution is only possible when $\sigma= \pm \sigma_{y}$. In the case of viscoplasticity as in Figure B.8, the parallel inclusion of the dashpot enables the element to carry more stress than that of the yield limit with the extra stress above $\sigma_{y}$ being taken up in the dashpot and consequently governing inelastic evolution. The extra stress, $\sigma_{e x}$, can be defined by

$$
\sigma_{e x}=\left\{\begin{array}{lll}
\sigma-\sigma_{y} & \text { if } \quad \sigma \geq \sigma_{y} \\
\sigma+\sigma_{y} & \text { if } \quad \sigma \leq-\sigma_{y} \\
0 & \text { if } \quad \sigma \in\left(-\sigma_{y}, \sigma_{y}\right)
\end{array}\right.
$$

For the case of nonzero extra stress, it can be shown that

$$
\sigma_{e x}=\left(|\sigma|-\sigma_{y}\right) \operatorname{sign}(\sigma)
$$

where $|\cdot|=\sqrt{(\cdot)^{2}}$ denotes the scalar magnitude and where sign $(\cdot)$ is a commonly used operator adequately defined by Simo and Hughes [61] as

$$
\operatorname{sign}(x)=\left\{\begin{array}{lll}
x & \text { if } & x>0 \\
-x & \text { if } & x<0
\end{array}\right.
$$

From (B.3), $\sigma_{e x}$ is nonzero only if

$$
f(\sigma)=|\sigma|-\sigma_{y}>0
$$

where $f(\sigma)$ is referred to as the yield function. To allow $\sigma_{e x}$ to be defined for all cases of $f(\sigma)$, following the work of Perić and Dettmer [46], Simo [59], Simo and Hughes [61], Zienkiewicz and Taylor [66] and Crisfield [8], a ramp function, $\langle\cdot\rangle$, is introduced such that

$$
\sigma_{e x}=\langle f(\sigma)\rangle \frac{\partial f(\sigma)}{\partial \sigma}
$$

where

$$
\langle x\rangle=\frac{(x+|x|)}{2}=\left\{\begin{array}{lll}
x & \text { if } & x>0 \\
0 & \text { if } & x \leq 0
\end{array}\right.
$$

and also it was convenient to exploit the differential of (B.6) where

$$
\frac{\partial}{\partial \sigma}(f(\sigma))=\frac{\partial}{\partial \sigma}\left(\sqrt{\sigma^{2}}-\sigma_{y}\right)=\frac{\sigma}{\sqrt{\sigma^{2}}}=\operatorname{sign}(\sigma)
$$

Now (B.7) is consistent with (B.3). 
Observing that the inelastic evolution of a dashpot is governed by the newtonian evolution equation similar to Equation 4.2 [17, 43, 61], then for the viscoplastic element

$$
\begin{gathered}
\dot{\varepsilon}^{i}=\frac{1}{\eta} \sigma_{e x}=\frac{\langle f(\sigma)\rangle}{\eta} \frac{\partial f(\sigma)}{\partial \sigma} \\
f(\sigma)=|\sigma|-\sigma_{y}
\end{gathered}
$$

A viscoplastic constitutive relationship in the form of (B.10) was first proposed by Perzyna [49].

The one-dimensional viscoplastic constitutive theory presented so far has dealt with perfect viscoplasticity in the sense that strain hardening of the plastic element (friction slider) has not been accounted for. Both isotropic and kinematic hardening have been shown to be important properties for semicrystalline polymers and so further development is necessary.

Firstly, the elastic limit, $\mathbb{E}_{\sigma}$, for the case of perfect plasticity is diagrammatically represented in Figure B.9. When stress is within the elastic limit, $|\sigma|-\sigma_{y} \leq 0$, deformation is wholly elastic. For stresses exceeding the elastic range, (B.6) is satisfied and there is inelastic evolution governed by (B.10).

When strain hardening is present, the internal variables $\alpha_{i s o}$ and $\alpha_{k i n}$ are introduced [61] which are related to the inelastic strain evolution by

$$
\begin{aligned}
& \dot{\alpha}_{i s o}=\left|\dot{\varepsilon}^{i}\right| \\
& \dot{\alpha}_{k i n}=\dot{\varepsilon}^{i}
\end{aligned}
$$

Graphical representation of the effect of isotropic and kinematic hardening on the elastic range in response to inelastic strain evolution is provided in Figures B.9 (b) and (c) respectively. The strain space internal variables $\alpha_{i s o}$ and $\alpha_{k i n}$ are related to the stress space elastic range through the isotropic and kinematic hardening moduli, $K$ and $H$.

Combining the boundary expressions of the elastic range from Figure B.9 (b) and (c) for the combined case of isotropic and kinematic hardening gives the new expression for yield function

$$
f\left(\sigma, \alpha_{i s o}, \alpha_{k i n}\right)=\left|\sigma-H \alpha_{k i n}\right|-\left(\sigma_{y}+K \alpha_{i s o}\right)
$$

Because (B.12) is expressed in a combination of stress and strain space terms, it is convenient from a numerical standpoint to introduce the stress space conjugates to the internal variables, $q_{i s o}$ and $q_{k i n}$, where

$$
\begin{aligned}
q_{i s o} & =-K \alpha_{i s o} \\
q_{k i n} & =-H \alpha_{k i n}
\end{aligned}
$$

MTDM_revision.tex; $15 / 05 / 2007 ; 8: 55 ;$ p.36 


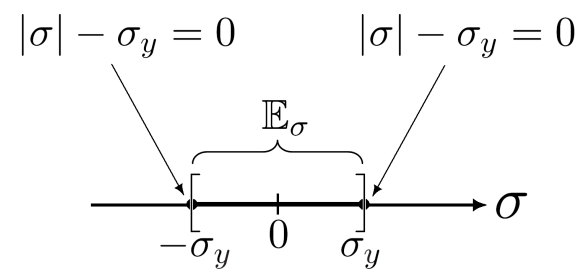

(a)

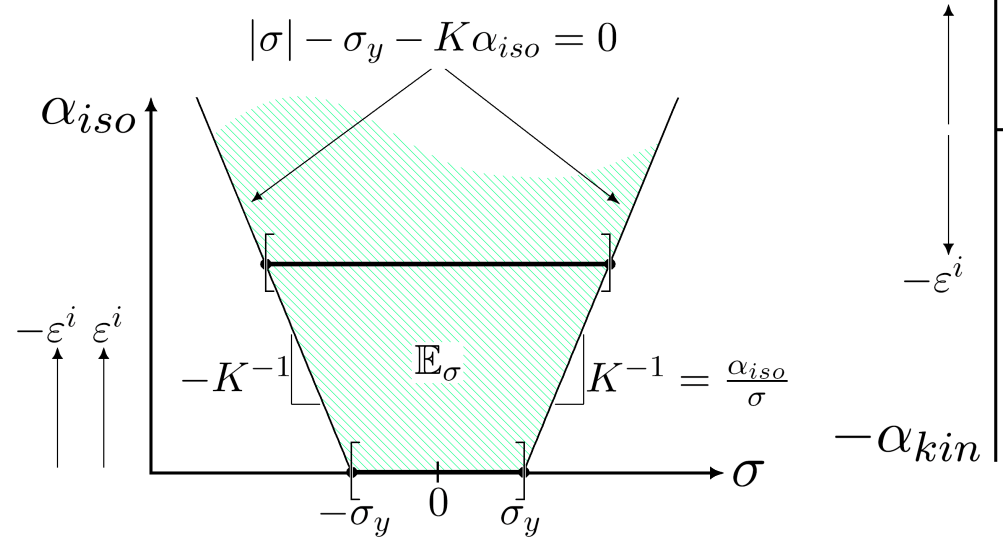

(b)

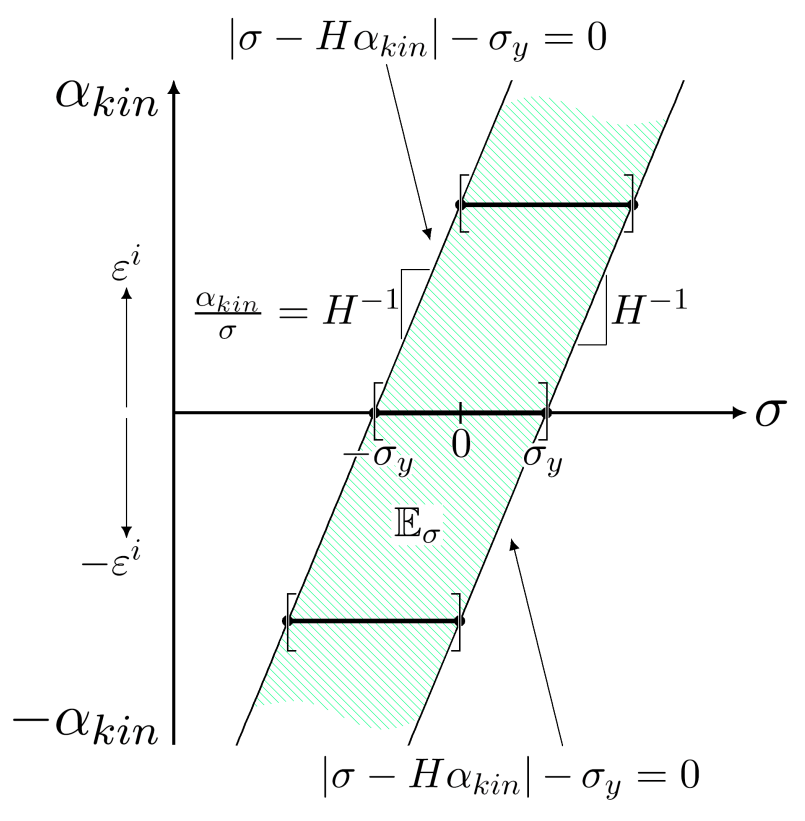

(c)

Figure B.9. Diagrammatical representation of the elastic limit for (a) perfect plasticity, (b) isotropic hardening (developed from Simo and Hughes [61], Figure 1.6, page 13) and (c) kinematic hardening.

such that now

$$
f\left(\sigma+q_{k i n}, q_{i s o}\right)=\left|\sigma+q_{k i n}\right|-\left(\sigma_{y}-q_{i s o}\right)
$$

An identical procedure to that carried out for the perfect viscoplastic case above then leads us to the result

$$
\dot{\varepsilon}^{i}=\frac{\left\langle f\left(\sigma+q_{k i n}, q_{i s o}\right)\right\rangle}{\eta} \frac{\partial f\left(\sigma+q_{k i n}, q_{i s o}\right)}{\partial \sigma}
$$

where now

$$
\frac{\partial f\left(\sigma+q_{k i n}, q_{i s o}\right)}{\partial \sigma}=\frac{\sigma+q_{k i n}}{\left|\sigma+q_{k i n}\right|}=\operatorname{sign}\left(\sigma+q_{k i n}\right)
$$

It is a useful result to derive (B.14) with respect to $q_{i s o}$ and $q_{k i n}$ such that

$$
\begin{aligned}
& \frac{\partial f\left(\sigma+q_{k i n}, q_{i s o}\right)}{\partial q_{\text {iso }}}=1 \\
& \frac{\partial f\left(\sigma+q_{k i n}, q_{i s o}\right)}{\partial q_{k i n}}=\frac{\sigma+q_{k i n}}{\left|\sigma+q_{k i n}\right|}=\frac{\partial f\left(\sigma+q_{k i n}, q_{i s o}\right)}{\partial \sigma}
\end{aligned}
$$


Now using (B.11), (B.17) and (B.18) and noting that $\left|\frac{x}{\sqrt{x^{2}}}\right|=1$, with some development the evolution of internal hardening variables can be defined by

$$
\dot{\alpha}_{i s o}=\frac{\left\langle f\left(\sigma+q_{k i n}, q_{i s o}\right)\right\rangle}{\eta} \frac{\partial f\left(\sigma+q_{k i n}, q_{i s o}\right)}{\partial q_{i s o}}
$$

and

$$
\dot{\alpha}_{k i n}=\frac{\left\langle f\left(\sigma+q_{k i n}, q_{i s o}\right)\right\rangle}{\eta} \frac{\partial f\left(\sigma+q_{k i n}, q_{i s o}\right)}{\partial q_{k i n}}
$$

A numerical solution strategy such as radial return mapping or closest point projection [61] can be used to solve for the mutually dependent plastic evolution equations, (B.15), (B.19) and (B.20). Equations (B.2), (B.14), (B.15), (B.19) and (B.20) constitute a complete constitutive equation set for one-dimensional viscoplasticity accounting for isotropic and kinematic hardening.

\section{References}

1. Bodner, S. R. and Y. Partom: 1972, 'A large deformation elastic-viscoplastic analysis of a thick-walled spherical shell'. J. Appl. Mech. 39, 751-757.

2. Bodner, S. R. and Y. Partom: 1975, 'Constitutive equations for elasticviscoplastic strain-hardening materials'. J. Appl. Mech. 42, 385-389.

3. Bonet, J. and R. D. Wood: 1997, Nonlinear continuum mechanics for finite element analysis. Melbourne: Cambridge University Press.

4. Brostow, W. and R. D. Corneliussen (eds.): 1986, Failure of Plastics. New York: Hanser Publishers.

5. Brusselle-Dupend, N., D. Lai, X. Feaugas, M. Guigon, and M. Clavel: 2001, 'Mechanical behavior of a semicrystalline polymer before necking. Part I: Characterization of uniaxial behavior'. Polym. Eng. Sci. 41, 66-76.

6. Brusselle-Dupend, N., D. Lai, X. Feaugas, M. Guigon, and M. Clavel: 2003, 'Mechanical behavior of a semicrystalline polymer before necking. Part II: Modeling of uniaxial behavior'. Polym. Eng. Sci. 43, 501-518.

7. Budinski, K. G. and M. K. Budinski: 2002, Engineering Materials: Properties and Selection. Sydney: Prentice Hall.

8. Crisfield, M. A.: 1997, Advanced Topics, Vol. 2 of Non-linear finite element analysis of solids and structures. Brisbane: John Wiley and Sons Ltd.

9. Drozdov, A. D.: 2001, 'Modeling the viscoelastoplastic behavior of amorphous glassy polymers'. Polym. Eng. Sci. 41, 1762-1770.

10. Drozdov, A. D., S. Agarwal, and R. K. Gupta: 2004, 'The effect of temperature on the viscoelastic behavior of linear low-density polyethylene'. Archive Appl. Mech. 73, 591-614.

11. Drozdov, A. D. and J. deC. Christiansen: 2003, 'Model for the viscoelastic and viscoplastic responses of semicrystalline polymers'. J. Appl. Polym. Sci. 88, $1438-1450$. 
12. Drozdov, A. D. and J. deC. Christiansen: 2004, 'Constitutive equations for the viscoplastic response of isotactic polypropylene in cyclic tests: The effect of strain rate'. Polym. Eng. Sci. 44, 548-556.

13. Drozdov, A. D. and A. L. Kalamkarov: 1996, 'A constitutive model for nonlinear viscoelastic behavior of polymers'. Polym. Eng. Sci. 36, 1907-1919.

14. Drozdov, A. D. and Q. Yuan: 2003, 'Effect of annealing on the viscoelastic and viscoplastic responses of low-density polyethylene'. J. Polym. Sci., Part B: Polym. Phys. 41, 1638-1655.

15. Duffo, P., B. Monasse, J. M. Haudin, C. G'Sell, and A. Dahoun: 1995, 'Rheology of polypropylene in the solid state'. J. Mater. Sci. 30, 701-711.

16. Eyring, H.: 1936, 'Viscosity, plasticity, and diffusion as examples of absolute reaction rates'. J. Chem. Phys. 4, 283-291.

17. Findley, W. N., J. S. Lai, and K. Onaran: 1976, Creep and relaxation of nonlinear viscoelastic materials. New York: Dover Publications, Inc.

18. G'Sell, C., A. Dahoun, V. Favier, J. M. Hiver, M. J. Philippe, and G. R. Canova: 1997, 'Microstructure transformation and stress-strain bahavior of isotactic polypropylene under large plastic deformation'. Polym. Eng. Sci. 37, 17021711.

19. G'Sell, C., A. Dahoun, F. X. Royer, and M. J. Philippe: 1999, 'The influence of the amorphous matrix on the plastic hardening at large strain of semicrystalline polymers'. Modelling Simul. Mater. Sci. Eng. 7, 817-828.

20. G'Sell, C. and J. J. Jonas: 1979, 'Determination of the plastic behaviour of solid polymers at constant true strain rate'. J. Mater. Sci. 14, 583-591.

21. G'Sell, C. and J. J. Jonas: 1981, 'Yield and transient effects during the plastic deformation of solid polymers'. J. Mater. Sci. 16, 1956-1974.

22. Haward, R. N., 'Strain hardening of thermoplastics'. Macromolecules.

23. Holzapfel, G. A.: 1996, 'On large strain viscoelasticity: Continuum formulation and finite element applications to elastomeric structures'. Int. J. Numer. Meth. Engng. 39, 3903-3926.

24. Holzapfel, G. A. and J. C. Simo: 1996, 'A new viscoelastic constitutive model for continuous media at finite thermomechanical changes'. Int. J. Solids Struct. 33, 3019-3034.

25. Hughes, T. J. R.: 1984, 'Numerical implementation of constitutive models: Rate-independent deviatoric plasticity'. In: S. Nemat-Nasser, R. Asaro, and G. Hegemier (eds.): Theoretical foundations for large scale computations of nonlinear material behaviour. Dordrecht, pp. 29-57.

26. Itskov, M.: 2000, 'On the theory of fourth-order tensors and their applications in computational mechanics'. Comput. Methods Appl. Mech. Engrg. 189, 419438.

27. Itskov, M.: 2001, 'A generalized orthotropic hyperelastic material model with application to incompressible shells'. Int. J. Numer. Meth. Engng. 50, 17771799 .

28. Itskov, M.: 2002, 'The derivative with respect to a tensor: some theoretical aspects and applications'. Z. Angew. Math. Mech. 82, 535-544.

29. Jr., G. H. H. J. W. and H. Eyring: 1945, 'Mechanical properties of textiles'. I. Text. Res. J. 15, 295-311.

30. Kintzel, O.: 2005, 'Fourth-order tensors - tensor differentiation with applications to continuum mechanics. Part II: Tensor analysis on manifolds'. Z. Angew. Math. Mech. 85, 1-23. 
31. Kitagawa, M. and T. Matsutani: 1988, 'Effect of time and temperature on nonlinear constitutive equation in polypropylene'. J. Mater. Sci. 23, 40854090.

32. Kitagawa, M., T. Mori, and T. Matsutani: 1989, 'Rate-dependent nonlinear constitutive equation for polypropylene'. J. Polym. Sci., Part B: Polym. Phys. 27, 85-95.

33. Kitagawa, M. and H. Takagi: 1990, 'Nonlinear constitutive equation for polyethylene under combined tension and torsion'. J. Polym. Sci., Part B: Polym. Phys. 28, 1943-1953.

34. Kletschkowski, T., U. Schomburg, and A. Bertram: 2004, 'An endochronic viscoplastic approach for materials with different behavior in tension and compression'. Mech. Time-Depend. Mat. 8, 119-135.

35. Kontou, E. and P. Farasoglou: 1998, 'Determination of the true stress-strain behaviour of polypropylene'. J. Mater. Sci. 33, 147-153.

36. Lai, J. and A. Bakker: 1995, 'An integral constitutive equation for nonlinear plasto-viscoelastic behavior of high-density polyethylene'. Polym. Eng. Sci. 35, 1339-1347.

37. Lifshitz, J. M. and H. Kolsky: 1967, 'Non-linear viscoelastic behaviour of polyethylene'. Int. J. Solids Structures 3, 383-397.

38. Nedjar, B.: 2002a, 'Frameworks for finite strain viscoelastic-plasticity based on multiplicative decompositions. Part I: Continuum formulations'. Comput. Methods Appl. Mech. Engng. 191, 1541-1562.

39. Nedjar, B.: 2002b, 'Frameworks for finite strain viscoelastic-plasticity based on multiplicative decompositions. Part II: Computational aspects'. Comput. Methods Appl. Mech. Engng. 191, 1563-1593.

40. Ogden, R. W.: 1984, Non-linear elastic deformations. New York: Dover Publications, Inc.

41. Oleynik, E. F.: 1990. In: E. Baer and A. Moet (eds.): High performance polymers: structure, properties, composites, fibres. Munich, p. 79.

42. Onaran, K. and W. N. Findley, 'Combined stress-creep experiments on a nonlinear viscoelastic material to determine the kernel functions for a multiple integral representation of creep'. Trans., Soc. Rheol. 9, 299-327.

43. Owen, D. R. J. and E. Hinton: 1986, Finite elements in plasticity: Theory and practice. Swansea: Pineridge Press Ltd.

44. Pegoretti, A., A. Guardini, C. Migliaresi, and T. Ricco: 2000, 'Recovery of post-yielding deformations in semicrystalline poly(ethylene-terephthalate)'. Polymer 41, 1857-1864.

45. Perić, D.: 1993, 'On a class of consitutive equations in viscoplasticity: Formulation and computational issues'. Int. J. Numer. Meth. Engng. 36, 1365-1393.

46. Perić, D. and W. Dettmer: 2003, 'A computational model for generalized inelastic materials at finite strains combining elastic, viscoelastic and plastic material behaviour'. Engng. Comp. 20, 768-787.

47. Perić, D. and D. R. J. Owen: 1992, 'A model for large deformations of elastoviscoplastic solids at finite strain: Computational issues'. In: D. Besdo and E. Stein (eds.): Finite inelastic deformations: Theory and applications. Berlin, pp. 299-312.

48. Perić, D. and D. R. J. Owen: 1998, 'Finite-element applications to the nonlinear mechanics of solids'. Rep. Prog. Phys. 61, 1495-1574.

49. Perzyna, P.: 1971, 'Thermodynamic theory of viscoplasticity'. In: C.-S. Yih (ed.): Advances in applied mechanics, Vol. 11. pp. 313-354. 
50. Popelar, C. F., C. H. Popelar, and V. H. Kenner: 1990, 'Viscoelastic material characterization and modeling for polyethylene'. Polym. Eng. Sci. 30, 577-586.

51. Quinson, R., J. Perez, M. Rink, and A. Pavan: 1996, 'Components of non-elastic deformation in amorphous glassy polymers'. J. Mater. Sci. 31, 4387-4394.

52. Reese, S. and S. Govindjee: 1998, 'A theory of finite viscoelasticity and numerical aspects'. Int. J. Solids Structures 35, 3455-3482.

53. Rosati, L. and N. Valoroso: 2004, 'A return map algorithm for general isotropic elasto/visco-plastic materials in principal space'. Int. J. Numer. Meth. Engng. 60, 461-498.

54. Schang, O., N. Billon, J. M. Muracciole, and F. Fernagut: 1996, 'Mechanical behavior of a ductile polyamide 12 during impact'. Polym. Eng. Sci. 36, 541550 .

55. Schapery, R. A.: 1997, 'Nonlinear viscoelastic and viscoplastic constitutive equations based on thermodynamics'. Mech. of Time-Depend. Mat. 1 pp. 209-240.

56. Schapery, R. A.: 1999, 'Nonlinear viscoelastic and viscoplastic constitutive equations with growing damage'. Int. J. Fracture. 97, 33-66.

57. Schrauwen, B. A. G., R. P. M. Janssen, L. E. Govaert, and H. E. H. Meijer: 2004, 'Intrinsic deformation behaviour of semicrystalline polymers'. Macromolecules 37, 6069-6078.

58. Simo, J. C.: 1987, 'On a fully three-dimensional finite-strain viscoelastic damage model: Formulation and computational aspects'. Comput. Methods Appl. Mech. Engrg. 60, 153-173.

59. Simo, J. C.: 1992, 'Algorithms for static and dynamic multiplicative plasticity that preserve the classical return mapping schemes of the infinitesimal theory'. Comput. Methods Appl. Mech. Engrg. 99, 61-112.

60. Simo, J. C. and T. J. R. Hughes: 1987, 'General return mapping algorithms for rate-independent plasticity'. In: C. S. Desai (ed.): Constitutive laws for engineering materials: Theory and applications. New York, pp. 221-231.

61. Simo, J. C. and T. J. R. Hughes: 2000, Computational Inelasticity. New York: Springer.

62. Simo, J. C. and R. L. Taylor: 1991, 'Quasi-incompressible finite elasticity in principal stretches. Continuum basis and numerical algorithms'. Comput. Methods Appl. Mech. Engrg. 85, 273-310.

63. Twizell, E. H. and R. W. Ogden: 1983, 'Non-linear optimization of the material constants in Ogden's stress-deformation function for incompressible isotropic elastic materials'. J. Austral. Math. Soc. Ser. B 24, 424-434.

64. Zhang, C. and I. D. Moore: 1997a, 'Nonlinear mechanical response of high density polyethylene. Part I: Experimental investigation and model evaluation'. Polym. Eng. Sci. 37, 404-413.

65. Zhang, C. and I. D. Moore: 1997b, 'Nonlinear mechanical response of high density polyethylene. Part II: Uniaxial constitutive modeling'. Polym. Eng. Sci. 37, 414-420.

66. Zienkiewicz, O. C. and R. L. Taylor: 2003, Solid Mechanics, Vol. 2 of The finite element method. Sydney: Butterworth Heinemann, 5 edition. 